\title{
A New Nonlinear Chaotic Complex Model and Its Complex Antilag Synchronization
}

\author{
Emad E. Mahmoud ${ }^{1,2}$ and Fatimah S. Abood ${ }^{3}$ \\ ${ }^{1}$ Department of Mathematics, College of Science, Sohag University, Sohag 82524, Egypt \\ ${ }^{2}$ Department of Mathematics, College of Science, Taif University, Taif, Saudi Arabia \\ ${ }^{3}$ Department of Mathematics, College of Science, King Khalid University, Abha, Saudi Arabia \\ Correspondence should be addressed to Emad E. Mahmoud; emad_eluan@yahoo.com
}

Received 4 April 2017; Revised 23 May 2017; Accepted 8 June 2017; Published 3 August 2017

Academic Editor: Olfa Boubaker

Copyright (c) 2017 Emad E. Mahmoud and Fatimah S. Abood. This is an open access article distributed under the Creative Commons Attribution License, which permits unrestricted use, distribution, and reproduction in any medium, provided the original work is properly cited.

\begin{abstract}
Another chaotic nonlinear Lü model with complex factors is covered here. We can build this riotous complex system when we add a complex nonlinear term to the third condition of the complex Lü system and think of it as if every one of the factors is mind boggling or complex. This system in real adaptation is a 6-dimensional continuous autonomous chaotic system. Different types of chaotic complex Lü system are developed. Also, another sort of synchronization is presented by us which is simple for anybody to ponder for the chaotic complex nonlinear system. This sort might be called a complex antilag synchronization (CALS). There are irregular properties for CALS and they do not exist in the literature; for example, (i) the CALS contains or fused two sorts of synchronizations (antilag synchronization ALS and lag synchronization LS); (ii) in CALS the attractors of the main and slave systems are moving opposite or similar to each other with time lag; (iii) the state variable of the main system synchronizes with a different state variable of the slave system. A scheme is intended to accomplish CALS of chaotic complex systems in light of Lyapunov function. The acquired outcomes and effectiveness can be represented by a simulation case for our new model.
\end{abstract}

\section{Introduction}

The chaotic system is an extremely specific nonlinear dynamical system. This chaotic system has numerous properties like the sensibility to starting conditions and in addition a sporadic, unusual conduct. The "butterfly effect" is the wellknown name of the sensibility of the introductory states of the chaotic systems [1]. Since the development of the first chaotic attractor in a three-dimensional (3D) self-governing chaotic system in 1963 by Lorenz [2], over the most recent 30 years chaos has been seriously explored [3-5]. Other chaotic systems were ordered in progression relying upon the arrangement of Lorenz, for example, Lü and Chen chaotic systems [6] and Liu chaotic system [7]. Each of the past chaotic systems has one positive Lyapunov exponent, and every one of them is three-dimensional chaotic system. Be that as it may, there are likewise many fascinating cases including complex factors which have not been effectively investigated. For instance, we say here the mind-boggling
Lorenz conditions which are utilized to depict and reproduce the physics of detuned laser and thermal convection of fluid flows [8] and some of its dynamical properties are contemplated in [9]. The electric field abundance and the nuclear polarization sufficiency are both complex; for details see [10] and reference therein. In secured communications, utilizing complex variable expands the substance security of the transmitted data [11]. Complex Lü and Chen systems are presented and contemplated as of late in [12].

In 2007, Mahmoud et al. [12] introduced the complex Lü system as follows:

$$
\begin{aligned}
& \dot{x}=\rho(y-x), \\
& \dot{y}=v y-x z, \\
& \dot{z}=\frac{1}{2}(\bar{x} y+x \bar{y})-\mu z,
\end{aligned}
$$

where $\rho, v$, and $\mu$ are positive parameters, $x=w_{1}+j w_{2}$ and $y=w_{3}+j w_{4}$ are complex components, $z=w_{5}$ is real variable, 
$j=\sqrt{-1}, w_{i}=1,2, \ldots, 5$, dots refer to derivatives according to time, and the meaning of overbar is complex conjugate variables.

Complex synchronization of chaotic (hyperchaotic) complex systems is a critical nonlinear occurrence [13-25]. Complex synchronization of chaotic complex systems considers a couple of complex chaotic systems called main and slave systems, and it means to accomplish asymptotic tracking of the conditions of the slave system to the conditions of the main system. In the quest for the higher ability for enhancing the security of communication systems, some efforts have been dedicated to synchronization with complex-esteemed scaling components between chaotic (hyperchaotic) complexvariable systems. Lately, complex complete synchronization (CCS) [13], complex lag synchronization (CLS) [14], complex projective synchronization (CPS) [15], and modified projective synchronization with complex scaling components (CMPS) $[16,17]$ are explored for coupled chaotic complex dynamical systems. Complex modified generalized projective synchronization (CMGPS) [18] and complex modified hybrid projective synchronization (CMHPS) [19] were executed between the same or different dimensional partial request complex chaotic (hyperchaotic). Complex generalized synchronization as for a mind-boggling vector map [20, 21] was presented for two indistinguishable or nonidentical chaotic (hyperchaotic) complex-variable systems. The complex modified projective synchronization of complex chaotic (hyperchaotic) systems with uncertain complex parameters was studied in $[22,23]$ while the complex modified function projective synchronization of complex chaotic systems is investigated in $[24,25]$.

As of late, a few sorts of synchronization with time lag were concentrated; for example, antilag synchronization (ALS), lag synchronization (LS), and modified projective lag synchronization (MPLS) of two riotous or hyperchaotic complex systems are investigated in [26-29]. In designing the applications, time delay always exists. For example, in the arrangement of telephone communication, the receiver hears the speaker voice at time $t$. This is the speaker voice or transmitter at time $t-\tau$ ( $\tau \geq 0$ and it is the lag time). In chaos communication, the time lag is the transmit flag that transmits to the receiver's end [26,27].

In this research, we introduce a modern chaotic model with complex components by embedding a complex nonlinear expression to the third equation of the complex Lü system (1) as

$$
\begin{aligned}
& \dot{x}=\rho(y-x), \\
& \dot{y}=v y-x z, \\
& \dot{z}=\frac{1}{2}(\bar{x} y+x \bar{y})-\mu z+j \operatorname{Im}(x) \operatorname{Re}(y),
\end{aligned}
$$

where $\rho, v$, and $\mu$ are positive parameters, $x=w_{1}+j w_{2}, y=$ $w_{3}+j w_{4}$, and $z=w_{5}+j w_{6}$ are complex functions, $\operatorname{Im}(x)$ is imaginary part of $x$ equal to $w_{2}$, and $\operatorname{Re}(y)$ is the real part of $y$ equal to $w_{3}$.

In addition, we present a novel sort of synchronization which we can name as complex antilag synchronization
(CALS). The term CALS can be dealt with as synchronizing among ALS and LS. ALS occurs between the real part of the main system and the imaginary part of a slave system, while LS occurs between a real part of the slave system and an imaginary part of the main system.

This paper is organized as follows: in the tracking section invariance, dissipation, fixed points, and their stability analysis of some points are contemplated. The complex comportment of system (2) can without much of a stretch be studied. As indicated by estimations of Lyapunov exponents, we can figure the scope of parameters qualities at the chaotic attractors numerically. We get the best classification of the progression of (2) by the signs of Lyapunov types. We get the Lyapunov dimension of (2). We develop different types of chaotic complex Lü systems in Section 3. In Section 4 a definition of CALS is acquainted and a scheme to achieve CALS of chaotic complex nonlinear systems is proposed. In Section 5, we study CALS of two indistinguishable chaotic complexes Lü systems (2) as a case of Section 4. Finally, we will find that the fundamental conclusions of our investigations are totalized in Section 6.

\section{Basic Properties of System (2)}

We study the basic dynamical analysis of our new system (2).

The real version of system (2) reads

$$
\begin{aligned}
& \dot{w}_{1}=\rho\left(w_{3}-w_{1}\right), \\
& \dot{w}_{2}=\rho\left(w_{4}-w_{2}\right), \\
& \dot{w}_{3}=v w_{3}-w_{1} w_{5}+w_{2} w_{6}, \\
& \dot{w}_{4}=v w_{4}-w_{1} w_{6}-w_{2} w_{5}, \\
& \dot{w}_{5}=w_{1} w_{3}+w_{2} w_{4}-\mu w_{5}, \\
& \dot{w}_{6}=-\mu w_{6}+w_{2} w_{3} .
\end{aligned}
$$
ing.

System (3) has many main dynamical qualities as track-

2.1. Symmetry and Invariance. In system (3), we notice that this system is invariant transformation: $\left(w_{1}, w_{2}, w_{3}, w_{4}, w_{5}\right.$, $\left.w_{6}\right) \Rightarrow\left(-w_{1},-w_{2},-w_{3},-w_{4}, w_{5}, w_{6}\right)$; therefore if $\left(w_{1}, w_{2}, w_{3}\right.$, $\left.w_{4}, w_{5}, w_{6}\right)$ is the solution of system (3), then $\left(-w_{1},-w_{2},-w_{3}\right.$, $\left.-w_{4}, w_{5}, w_{6}\right)$ is known as the solution of the similar system.

2.2. Dissipation. It is clear to find $\nabla \cdot V=\sum_{l=1}^{6}\left(\partial \dot{w}_{i} / \partial w_{i}\right)=$ $\partial \dot{w}_{1} / \partial w_{1}+\partial \dot{w}_{2} / \partial w_{2}+\partial \dot{w}_{3} / \partial w_{3}+\partial \dot{w}_{4} / \partial w_{4}+\partial \dot{w}_{5} / \partial w_{5}+$ $\partial \dot{w}_{6} / \partial w_{6}=-2 \rho+2 v-2 \mu<0$. When $v<(\rho+\mu)$, the system is dissipative and meets $d V / d t=e^{-(v-\rho-\mu) t}$ with type shape. It implies that the volume component $V_{0}$ contracts to the volume component $V_{0} e^{-(v-\rho-\mu) t}$ at the time $t$. At the point when $t \rightarrow \infty$, each volume component which contains the system direction congregates to 0 with exponent rate form $v-\rho-\mu$. Thus, the majority of the system directions will finally be limited to zero volume subset, and the dynamic development is fixed on an attractor. 
2.3. Equilibria and Their Stability. The equilibria of system (3) can be found by solving the tracking system of equations:

$$
\begin{aligned}
& 0=\rho\left(w_{3}-w_{1}\right), \\
& 0=\rho\left(w_{4}-w_{2}\right), \\
& 0=v w_{3}-w_{1} w_{5}+w_{2} w_{6}, \\
& 0=v w_{4}-w_{1} w_{6}-w_{2} w_{5}, \\
& 0=w_{1} w_{3}+w_{2} w_{4}-\mu w_{5}, \\
& 0=-\mu w_{6}+w_{2} w_{3} .
\end{aligned}
$$

Obviously, $F_{0}=(0,0,0,0,0,0)$ is trivial fixed point. Other nonzero equilibria are given by

$$
\begin{aligned}
& F_{1}=(0, \sqrt{\mu v}, 0, \sqrt{\mu v}, v, 0), \\
& F_{2}=(0,-\sqrt{\mu v}, 0,-\sqrt{\mu v}, v, 0), \\
& F_{3}=(\sqrt{\mu v}, 0, \sqrt{\mu v}, 0, v, 0), \\
& F_{4}=(-\sqrt{\mu v}, 0,-\sqrt{\mu v}, 0, v, 0), \\
& F_{5}=(\sqrt{\mu v}, j \sqrt{\mu v}, \sqrt{\mu v}, j \sqrt{\mu v}, 0, j v), \\
& F_{6}=(\sqrt{\mu v},-j \sqrt{\mu v}, \sqrt{\mu v},-j \sqrt{\mu v}, 0,-j v), \\
& F_{7}=(-\sqrt{\mu v}, j \sqrt{\mu v},-\sqrt{\mu v}, j \sqrt{\mu v}, 0,-j v), \\
& F_{8}=(-\sqrt{\mu v},-j \sqrt{\mu v},-\sqrt{\mu v},-j \sqrt{\mu v}, 0, j v) .
\end{aligned}
$$

2.3.1. Stability of $F_{0}$. To study the stability of $F_{0}$ the Jacobian matrix of system (3) at $F_{0}$ is

$$
J_{F_{0}}=\left(\begin{array}{cccccc}
-\rho & 0 & \rho & 0 & 0 & 0 \\
0 & -\rho & 0 & \rho & 0 & 0 \\
0 & 0 & v & 0 & 0 & 0 \\
0 & 0 & 0 & v & 0 & 0 \\
0 & 0 & 0 & 0 & -\mu & 0 \\
0 & 0 & 0 & 0 & 0 & -\mu
\end{array}\right) .
$$

The characteristic polynomial equation is

$$
(\lambda+\rho)^{2}(\lambda+\mu)^{2}(\lambda-v)^{2}=0 .
$$

Then the eigenvalues are $\lambda_{1}=\lambda_{2}=-\rho, \lambda_{3}=\lambda_{4}=-\mu$, and $\lambda_{5}=\lambda_{6}=v$. The trivial fixed point $(0,0,0,0,0,0)$ is a stable point if $v$ has negative values and $\rho$ and $\mu$ are positive and else, it is not stable fixed point.

2.3.2. Stability of $F_{1}, F_{2}, F_{3}$, and $F_{4} . F_{1}, F_{2}, F_{3}$, and $F_{4}$ have the same characteristic polynomial, which is

$$
\begin{aligned}
& {\left[\lambda^{3}+\lambda^{2}(\mu-\nu+\rho)+\lambda \mu \rho+2 \mu v \rho\right]} \\
& \cdot\left[\lambda^{3}+\lambda^{2}(\mu-\nu+\rho)+\lambda \mu(\rho-v)+\mu v \rho\right]=0 .
\end{aligned}
$$

According to the Routh-Hurwitz theorem [30] the necessary and sufficient conditions for all roots to have negative real parts are if and only if (stable fixed points)

$$
\begin{aligned}
v & <\rho+\mu, \\
\mu v \rho & >0, \\
3 v & <\rho+\mu, \\
\mu\left(1-\frac{v}{\rho}\right)+\rho+\frac{v^{2}}{\rho} & >3 v ;
\end{aligned}
$$

otherwise they are unstable fixed points.

Likewise we can study the stability of $F_{5}, F_{6}, F_{7}$, and $F_{8}$.

2.4. Lyapunov Exponents. System (3) in vector notation can be written as

$$
\dot{w}(t)=g(w(t) ; M),
$$

where $w(t)=\left(w_{1}(t), w_{2}(t), w_{3}(t), w_{4}(t), w_{5}(t), w_{6}(t)\right)^{T}$ is the state space vector, $g=\left(g_{1}, g_{2}, g_{3}, g_{4}, g_{5}, g_{6}\right)^{T}, M$ is the set of parameters, and $(\cdots)^{T}$ indicates transpose. The equations for small deviations $\delta w$ from the trajectory $w(t)$ are

$$
\delta \dot{w}(t)=J_{k I}(w(t) ; M) \delta w, \quad k=I=1,2,3,4,5,6,
$$

where $J_{k I}=\partial g_{j} / \partial w_{I}$ is the Jacobian matrix of the form

$$
J_{k I}=\left(\begin{array}{cccccc}
-\rho & 0 & \rho & 0 & 0 & 0 \\
0 & -\rho & 0 & \rho & 0 & 0 \\
-w_{5} & w_{6} & v & 0 & -w_{1} & w_{2} \\
-w_{6} & -w_{5} & 0 & v & -w_{2} & -w_{1} \\
w_{3} & w_{4} & w_{1} & w_{2} & -\mu & 0 \\
0 & w_{3} & w_{2} & 0 & 0 & -\mu
\end{array}\right) .
$$

The Lyapunov exponents $l_{I}$ of the system are defined by [31]

$$
l_{I}=\lim _{t \rightarrow \infty} \frac{1}{t} \log \frac{\left\|\partial w_{I}(t)\right\|}{\left\|\partial w_{I}(0)\right\|} .
$$

To find $l_{I}$, (10) and (11) must be numerically determined simultaneously. Runge-Kutta method of order 4 is used to compute $l_{I}$.

For the case of $\rho=21, v=10$, and $\mu=6$, the initial conditions are as follows: $t_{0}=0, w_{1}(0)=1, w_{2}(0)=2$, $w_{3}(0)=3, w_{4}(0)=4, w_{5}(0)=5$, and $w_{6}(0)=6$. We compute the Lyapunov exponents as $l_{1}=2.034, l_{2}=0, l_{3}=-0.99$, $l_{4}=-4.16, l_{5}=-22.49$, and $l_{6}=-2.45$.

This implies that our system (3) under this selection of $\rho, v, \mu$ is a chaotic system due to the fact that one of the Lyapunov exponents is positive.

The Lyapunov dimension of the attractors of (3) according to Kaplan-Yorke conjecture is defined as [32]

$$
D=\Omega+\frac{\sum_{k=1}^{\Omega} l_{k}}{\left|l_{\Omega+1}\right|},
$$

such that $\Omega$ is the largest integer that $\sum_{k=1}^{\Omega} l_{k}>0$ and $\sum_{k=1}^{\Omega+1} l_{k}<0$. The Lyapunov dimension of this chaotic attractor using (14) with $\rho=21, v=10$, and $\mu=6$ is $D=3.2509$. 


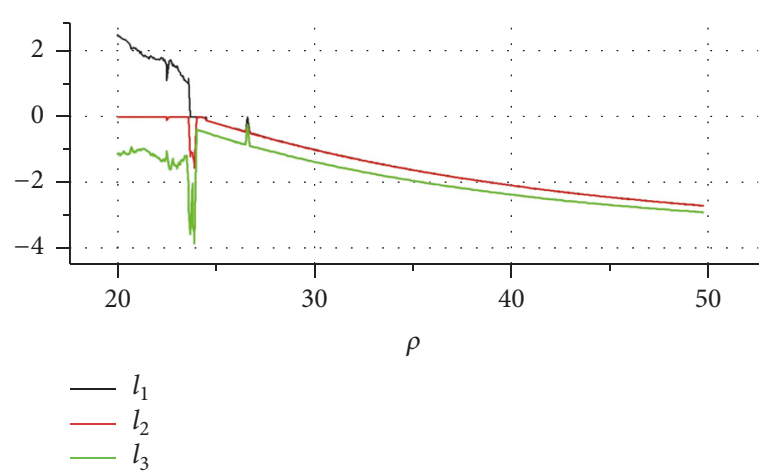

(a)

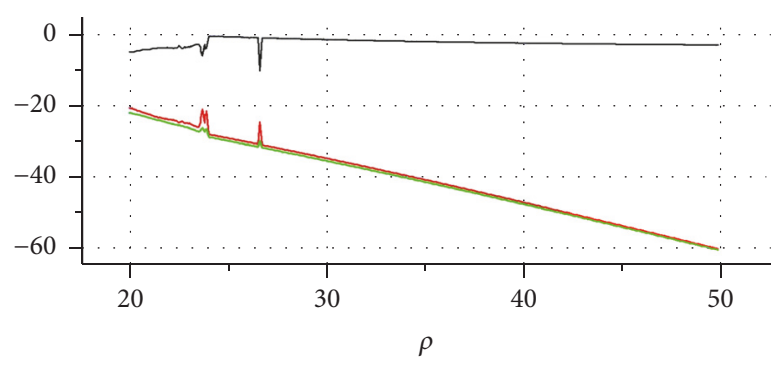

$-l_{4}$
$-l_{5}$
$-l_{6}$

(b)

Figure 1: The Lyapunov exponents $l_{I}, I=1,2, \ldots, 6$ of system (3) when we fix $v=10$ and $\mu=6$ and vary $\rho \in[20,50]$.

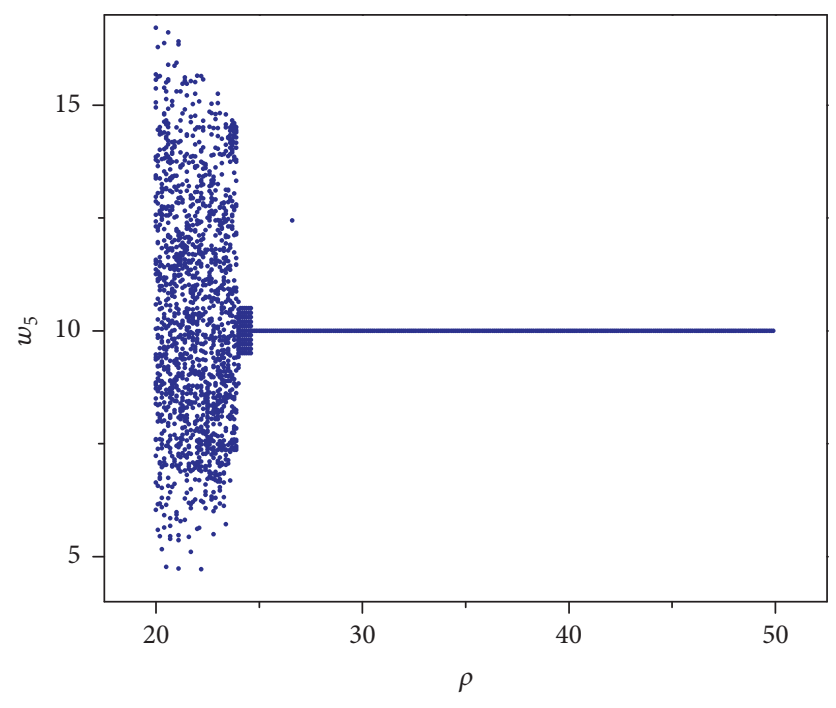

FIGURE 2: The bifurcation diagram of system (3) when the parameter $\rho$ is varying $\rho \in[20,50]$.

2.4.1. Fix $v=10$ and $\mu=6$ and Vary $\rho \in[20,50]$. In light of Lyapunov exponents $l_{I}$ (13) the system' parameter qualities were computed (3) at which chaotic attractors, periodic, quasiperiodic attractors and fixed points exist. The review modifies and differs one parameter and fixes the extra parameters as indicated by their states which fulfill the dissipative condition and the dependability state of the trifling fixed focuses.

Utilizing (13) we ascertain $l_{I}, I=1,2, \ldots, 6$, and the estimations of $l_{1}, l_{2}, \ldots, l_{6}$ versus $\rho$ are plotted in Figure 1.

Obviously from Figure 1, when $\rho \in[20,23.6]$ system (3) has chaotic attractors. The intermittent or periodic attractors of (3) exist for $\rho$ which lies in the interims $(23.6,24]$. The quasiperiodic conduct models up when $\rho \in(24.1,24.6]$ while the arrangement is equilibrium point when $\rho \in(24.6,50]$.

In Figure 2, we figure the bifurcation diagram of system (3) when the parameter $\rho$ is varying or modifying to guarantee that our system is chaotic. A bifurcation diagram outlines a system parameter on the horizontal axis and a report of the attractor's action on the vertical axis. So, bifurcation diagrams present a kind method to picture how a system's behavior varies according to the value of a parameter [33]. Figure 2 shows $\left(\rho, w_{5}\right)$ bifurcation diagram for $\rho \in[20,50]$. It can be discerned that when $\rho \in[20,23.6]$, system (3) has solutions that approach chaotic attractors and when $\rho \in$ $(23.6,24.6]$ it has periodic and quasiperiodic behavior. The equilibrium points are shown when $\rho \in(24.6,50]$. The dynamical behavior of Figure 2 is similar to this which is given in Figure 1.

Testing this numerically (utilizing, e.g., Mathematica 7 programming) in different sections and best notes is found and is agreeable with our outcomes. For instance, picking $v=10$ and $\mu=6$, with the underlying conditions $t_{0}=0$, $w_{1}(0)=1, w_{2}(0)=2, w_{3}(0)=3, w_{4}(0)=4, w_{5}(0)=5$, and $w_{6}(0)=6$, and when $\rho=21$, the arrangement of system (3) has chaotic attractor (see Figure 3(a)). In Figure 3(b) the arrangement is periodic with $\rho=23.9$. The quasiperiodic attractor is represented in Figure 3(c), $\rho=24$.2. In Figure 3(d) the balance point is indicated when $\rho=30$.

\section{Different Forms of Chaotic Complex Lü Systems}

In this section, we demonstrate that the complex Lü system (2) is not just the one that has chaotic attractors with all factors or variables being complex. In this way, we search for different systems that yield chaotic conduct. We can build these systems in light of system (1) by adding a complex nonlinear term to the third equation of (1) with thought, all factors are complex. We can build six different types of riotous complex Lü systems and compute their Lyapunov exponents as

$$
\begin{aligned}
& \dot{x}=\rho(y-x), \\
& \dot{y}=v y-x z, \\
& \dot{z}=\frac{1}{2}(\bar{x} y+x \bar{y})-\mu z+j \operatorname{Re}(x) \operatorname{Re}(y),
\end{aligned}
$$




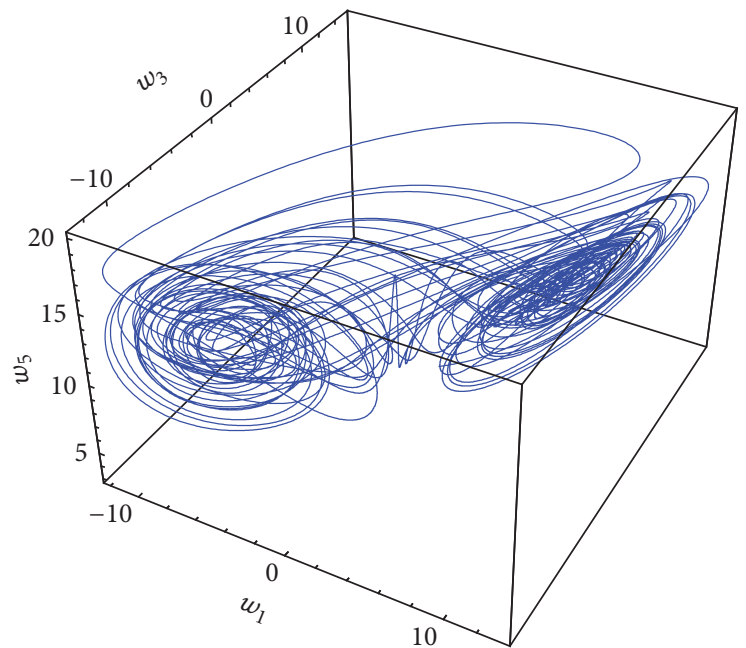

(a)

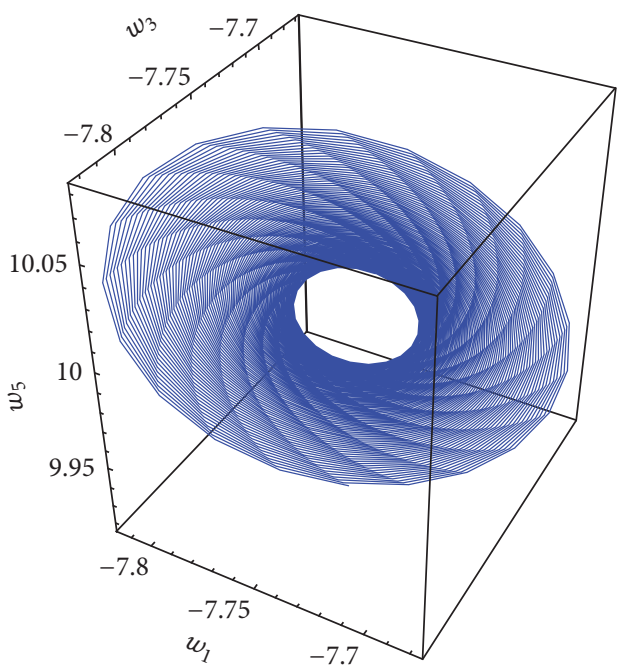

(c)

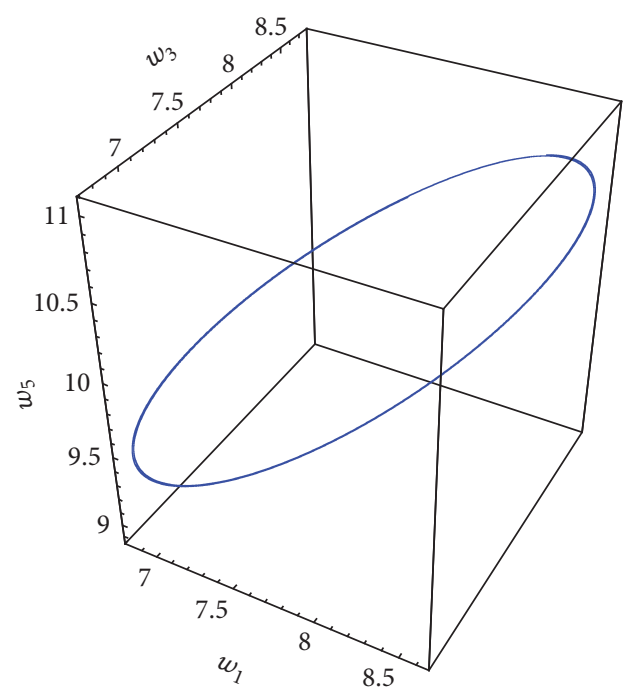

(b)

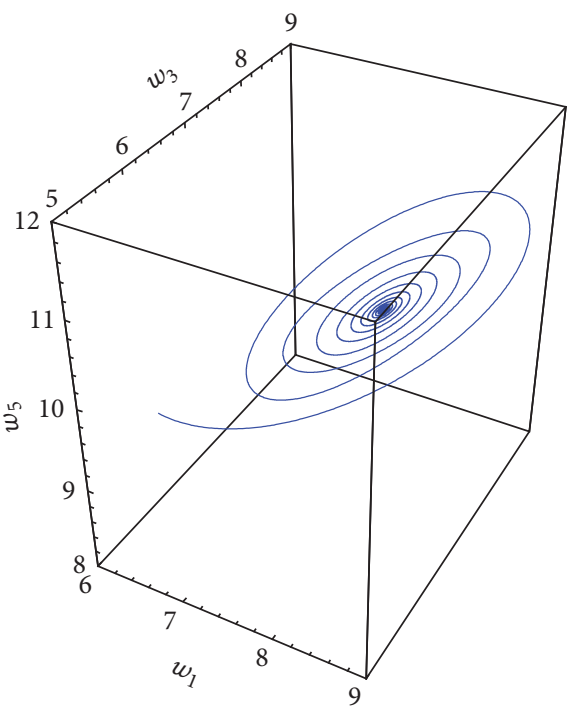

(d)

Figure 3: For $v=10$ and $\mu=6$, vary $\rho$. (a) Chaotic attractor, $\rho=21$ in $\left(w_{1}, w_{3}, w_{5}\right)$. (b) Periodic attractor, $\rho=23.9$ in $\left(w_{1}, w_{3}, w_{5}\right)$. (c) Quasiperiodic attractor, $\rho=24.2$ in $\left(w_{1}, w_{3}, w_{5}\right)$. (d) Fixed point, $\rho=30$ in $\left(w_{1}, w_{3}, w_{5}\right)$.

where $l_{1}=0.549, l_{2}=0, l_{3}=0, l_{4}=-4.19, l_{5}=-11.540$, $l_{6}=-17.72, \rho=20, \mu=2.5$, and $v=18$.

$$
\begin{aligned}
& \dot{x}=\rho(y-x), \\
& \dot{y}=v y-x z, \\
& \dot{z}=\frac{1}{2}(\bar{x} y+x \bar{y})-\mu z+j \operatorname{Im}(x) \operatorname{Im}(y),
\end{aligned}
$$

where $l_{1}=1.31, l_{2}=0, l_{3}=0, l_{4}=-5.804, l_{5}=-17.201$, $l_{6}=-22.013, \rho=25, \mu=3$, and $v=12$.

$$
\begin{aligned}
& \dot{x}=\rho(y-x), \\
& \dot{y}=v y-x z, \\
& \dot{z}=\frac{1}{2}(\bar{x} y+x \bar{y})-\mu z+j \operatorname{Im}(y) \operatorname{Re}(z),
\end{aligned}
$$

where $l_{1}=0.6, l_{2}=0, l_{3}=0, l_{4}=-4.88, l_{5}=-8.5, l_{6}=$ $-11.65, \rho=19, \mu=2.5$, and $v=16$.

$$
\begin{aligned}
& \dot{x}=\rho(y-x), \\
& \dot{y}=v y-x z, \\
& \dot{z}=\frac{1}{2}(\bar{x} y+x \bar{y})-\mu z+j \operatorname{Im}(z) \operatorname{Re}(y),
\end{aligned}
$$

where $l_{1}=0.957, l_{2}=0, l_{3}=0, l_{4}=-.98, l_{5}=-7.86, l_{6}=$ -13.7, $\rho=22, \mu=4$, and $v=18$.

$$
\begin{aligned}
& \dot{x}=\rho(y-x), \\
& \dot{y}=v y-x z, \\
& \dot{z}=\frac{1}{2}(\bar{x} y+x \bar{y})-\mu z+j \operatorname{Re}(z) \operatorname{Re}(y),
\end{aligned}
$$


where $l_{1}=1.200, l_{2}=0.01, l_{3}=0, l_{4}=-2.89, l_{5}=-10.96$, $l_{6}=-15.04, \rho=27, \mu=5$, and $v=14.6$.

$$
\begin{aligned}
& \dot{x}=\rho(y-x), \\
& \dot{y}=v y-x z, \\
& \dot{z}=\frac{1}{2}(\bar{x} y+x \bar{y})-\mu z+j \operatorname{Im}(x) \operatorname{Re}(z),
\end{aligned}
$$

where $l_{1}=1.6, l_{2}=0, l_{3}=0, l_{4}=-5.80, l_{5}=-16.908$, $l_{6}=-19.20, \rho=20, \mu=3$, and $v=11$.

All the above systems have one positive Lyapunov exponent. We ascertain these Lyapunov types as we accomplished in system (2) indistinguishable beginning conditions (Figure 1). The fundamental properties of these systems can be likewise examined as we have in Section 2 .

\section{A Definition and Achievement for CALS}

Consider the chaotic complex nonlinear system as follows:

$$
\dot{\mathbf{x}}=\mathbf{\Psi} \mathbf{x}+\mathbf{F}(\mathbf{x})
$$

where $\mathbf{x}=\left(x_{1}, x_{2}, \ldots, x_{n}\right)^{T} \in c^{n}$ is a state complex vector, $\mathbf{x}=\mathbf{x}^{\operatorname{Re}}+j \mathbf{x}^{\operatorname{Im}}, \mathbf{x}^{\operatorname{Re}}=\left(w_{1}, w_{3}, \ldots, w_{2 n-1}\right)^{T}, \mathbf{x}^{\operatorname{Im}}=\left(w_{2}, w_{4}, \ldots\right.$, $\left.w_{2 n}\right)^{T}, j=\sqrt{-1}, T$ denotes transpose, $\Psi \in R^{n \times n}$ is real (or complex) matrix of system parameters, $\mathbf{F}=\left(f_{1}, f_{2}, \ldots, f_{n}\right)^{T}$ is a vector of nonlinear complex functions, and coordinating Re and Im symbolize the real and imaginary parts of the state complex vector $\mathbf{x}$.

In this work we present and study the definition of CALS of two systems of shape (21) (indistinguishable) with known parameters by scheming a control plot. We tried its rightness numerically.

We consider two indistinguishable chaotic complex nonlinear systems of shape (21): one is the main system (we indicate the main system with the subscript $m$ ) as

$$
\dot{\mathbf{x}}_{m}=\dot{\mathbf{x}}_{m}^{\mathrm{Re}}+j \dot{\mathbf{x}}_{m}^{\operatorname{Im}}=\boldsymbol{\Psi} \mathbf{x}_{m}+\mathbf{F}\left(\mathbf{x}_{m}\right),
$$

and the second is the controlled slave system (with subscript s) as

$$
\dot{\mathbf{x}}_{s}=\dot{\mathbf{x}}_{s}^{\operatorname{Re}}+j \dot{\mathbf{x}}_{s}^{\operatorname{Im}}=\mathbf{\Psi} \mathbf{x}_{s}+\mathbf{F}\left(\mathbf{x}_{s}\right)+\mathbf{L},
$$

where the additive complex controllers are as follows: $\mathbf{L}=$ $\left(\mathbf{L}_{1}, \mathbf{L}_{2}, \ldots, \mathbf{L}_{n}\right)^{T}=\mathbf{L}^{\operatorname{Re}}+j \mathbf{L}^{\operatorname{Im}}, \mathbf{L}^{\operatorname{Re}}=\left(\zeta_{1}, \zeta_{3}, \ldots, \zeta_{2 n-1}\right)^{T}$, and $\mathbf{L}^{\operatorname{Im}}=\left(\zeta_{2}, \zeta_{4}, \ldots, \zeta_{2 n}\right)^{T}$.

Definition 1. Two indistinguishable complex dynamical systems coupled in a main-slave configuration can exhibit CALS if there exists a vector of the complex error function e defined as

$$
\mathbf{e}=\mathbf{e}^{\mathrm{Re}}+j \mathbf{e}^{\operatorname{Im}}=\lim _{t \rightarrow \infty}\left\|\mathbf{x}_{s}(t)+j \mathbf{x}_{m}(t-\tau)\right\|=0,
$$

where $\mathbf{e}=\left(e_{1}, e_{2}, \ldots, e_{n}\right)^{T}, \mathbf{x}_{m}$ and $\mathbf{x}_{s}$ are the state complex vectors of the main and slave, respectively, $\mathrm{e}^{\mathrm{Re}}=$ $\lim _{t \rightarrow \infty}\left\|\mathbf{x}_{s}^{\mathrm{Re}}(t)-\mathbf{x}_{m}^{\operatorname{Im}}(t-\tau)\right\|=0, \mathbf{e}^{\operatorname{Im}}=\lim _{t \rightarrow \infty} \| \mathbf{x}_{s}^{\operatorname{Im}}(t)+\mathbf{x}_{m}^{\mathrm{Re}}(t-$ $\tau) \|=0, \mathrm{e}^{\mathrm{Re}}=\left(e_{w_{1}}, e_{w_{3}}, \ldots, e_{w_{2 n-1}}\right)^{T}, \mathrm{e}^{\mathrm{Im}}=\left(e_{w_{2}}, e_{w_{4}}, \ldots\right.$, $\left.e_{w_{2 n}}\right)^{T}$, and $\tau$ is the positive time lag.
In CALS we define the error in simple case:

$x_{s}(t)+j x_{m}(t-\tau)=0$, as $t \rightarrow \infty$, where $x=w_{1}+j w_{2}$

$\left[w_{1 s}(t)+j w_{2 s}(t)\right]+j\left[w_{1 m}(t-\tau)+j w_{2 m}(t-\tau)\right]=0$,

$\left[w_{1 s}(t)+j w_{2 s}(t)\right]+\left[-w_{2 m}(t-\tau)+j w_{1 m}(t-\tau)\right]=0$,

$\left[w_{1 s}(t)-w_{2 m}(t-\tau)\right]+j\left[w_{2 s}(t)+w_{1 m}(t-\tau)\right]=0$,

$\Longrightarrow w_{1 s}(t)-w_{2 m}(t-\tau)=0 \Longrightarrow(\mathrm{LS})$

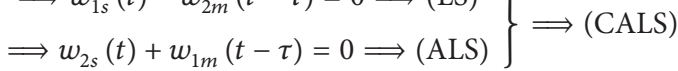

Box 1: Complex antilag synchronization (CALS).

$$
\begin{aligned}
& \text { In ALS the error in simple case: } \\
& x_{s}(t)+x_{m}(t-\tau)=0 \text {, as } t \rightarrow \infty \text {, where } x=w_{1}+j w_{2} \\
& {\left[w_{1 s}(t)+j w_{2 s}(t)\right]+\left[w_{1 m}(t-\tau)+j w_{2 m}(t-\tau)\right]=0,} \\
& {\left[w_{1 s}(t)+j w_{2 s}(t)\right]+\left[w_{1 m}(t-\tau)+j w_{2 m}(t-\tau)\right]=0,} \\
& {\left[w_{1 s}(t)+w_{1 m}(t-\tau)\right]+j\left[w_{2 s}(t)+w_{2 m}(t-\tau)\right]=0,} \\
& \Longrightarrow w_{1 s}(t)+w_{1 m}(t-\tau)=0 \Longrightarrow \text { (ALS) }
\end{aligned}
$$

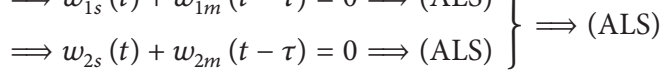

Box 2: Antilag synchronization (ALS) [27].

Remark 2. When $\tau=0$ in (24) we define complex antisynchronization CAS between (22) and (23).

Remark 3. If we define $\mathbf{e}=\lim _{t \rightarrow \infty}\left\|\mathbf{x}_{s}(t)-j \mathbf{x}_{m}(t-\tau)\right\|$ and $\tau=0$, we get CCS of systems (22) and (23) [13], while if $\tau>0$, we obtain CLS of the same systems [14].

Remark 4. The complex state is as follows:

$$
j=\rho(\cos \theta+j \sin \theta),
$$

where $\rho=|j|=1$ ( $\rho$ is the modulus of $j)$ and $\theta=\pi / 2(\theta$ is the phase of $j$ ).

Remark 5. The sum of the imaginary part of slave system $\mathbf{x}_{s}^{\operatorname{Im}}(t)$ and the real part of main system $\mathbf{x}_{m}^{\mathrm{Re}}(t-\tau)$ is vanishing when $t \rightarrow \infty$ (ALS) [27].

Remark 6. The error between the real part of slave system $\mathbf{x}_{s}^{\mathrm{Re}}(t)$ and the imaginary part of main system $\mathbf{x}_{m}^{\operatorname{Im}}(t-\tau)$ goes to zero as $t \rightarrow \infty$ (LS) [26].

Remark 7. The difference between CALS and ALS [27] can be illustrated from Boxes 1 and 2.

Theorem 8. If nonlinear controller is designed as

$$
\begin{aligned}
\mathbf{L}= & \mathbf{L}^{\mathrm{Re}}+j \mathbf{L}^{\mathrm{Im}}=-\boldsymbol{\Psi} \mathbf{x}_{s}(t)-\mathbf{F}\left(\mathbf{x}_{s}(t)\right) \\
& -j\left[\Psi \mathbf{x}_{m}(t-\tau)+\mathbf{F}\left(\mathbf{x}_{m}(t-\tau)\right)\right]-\xi \mathbf{e}, \\
& =-\boldsymbol{\Psi} \mathbf{x}_{s}^{\operatorname{Re}}(t)-\mathbf{F}^{\operatorname{Re}}\left(\mathbf{x}_{s}(t)\right)+\Psi \mathbf{x}_{m}^{\operatorname{Im}}(t-\tau) \\
& +\mathbf{F}^{\operatorname{Im}}\left(\mathbf{x}_{m}(t-\tau)\right)-\xi \mathbf{e}^{\operatorname{Re}}+j\left[-\Psi \mathbf{x}_{s}^{\operatorname{Im}}(t)\right.
\end{aligned}
$$




$$
\begin{aligned}
& -\mathbf{F}^{\mathrm{Im}}\left(\mathbf{x}_{s}(t)\right)-\Psi \mathbf{x}_{m}^{\mathrm{Re}}(t-\tau)-\mathbf{F}^{\mathrm{Re}}\left(\mathbf{x}_{\mathbf{m}}(t-\tau)\right) \\
& \left.-\xi \mathbf{e}^{\mathrm{Im}}\right]
\end{aligned}
$$

then the main system (22) and the slave system (23) are moving in CALS manner asymptotically, $\xi>0$.

Proof. From the definition of CALS,

$$
\mathbf{e}=\mathbf{e}^{\mathrm{Re}}+j \mathbf{e}^{\mathrm{Im}}=\mathbf{x}_{s}(t)+j \mathbf{x}_{m}(t-\tau) .
$$

So

$$
\begin{aligned}
\dot{\mathbf{e}} & =\dot{\mathbf{e}}^{\mathrm{Re}}+j \dot{\mathrm{e}}^{\operatorname{Im}}=\dot{\mathbf{x}}_{s}(t)+j \dot{\mathbf{x}}_{m}(t-\tau), \\
\dot{\mathbf{e}} & =\dot{\mathbf{e}}^{\operatorname{Re}}+j \dot{\mathbf{e}}^{\operatorname{Im}} \\
& =\dot{\mathbf{x}}_{s}^{\operatorname{Re}}(t)-\dot{\mathbf{x}}_{m}^{\operatorname{Im}}(t-\tau)+j\left[\dot{\mathbf{x}}_{s}^{\operatorname{Im}}(t)+\dot{\mathbf{x}}_{m}^{\operatorname{Re}}(t-\tau)\right],
\end{aligned}
$$

and from chaotic complex systems (22) and (23), we get the error complex dynamical system as follows:

$$
\begin{aligned}
\dot{\mathbf{e}} & =\dot{\mathbf{e}}^{\mathrm{Re}}+j \dot{\mathbf{e}}^{\mathrm{Im}}=\boldsymbol{\Psi} \mathbf{x}_{s}^{\mathrm{Re}}(t)+\mathbf{F}^{\mathrm{Re}}\left(\mathbf{x}_{s}(t)\right)-\boldsymbol{\Psi} \mathbf{x}_{m}^{\operatorname{Im}}(t \\
& -\tau)-\mathbf{F}^{\operatorname{Im}}\left(\mathbf{x}_{\mathbf{m}}(t-\tau)\right)+\mathbf{L}^{\mathrm{Re}}+j\left[\Psi \mathbf{x}_{s}^{\operatorname{Im}}(t)\right. \\
& +\mathbf{F}^{\operatorname{Im}}\left(\mathbf{x}_{s}(t)\right)+\Psi \mathbf{x}_{m}^{\operatorname{Re}}(t-\tau)+\mathbf{F}^{\operatorname{Re}}\left(\mathbf{x}_{m}(t-\tau)\right) \\
& \left.+\mathbf{L}^{\operatorname{Im}}\right] .
\end{aligned}
$$

By separating the real and the imaginary parts in (29), the error complex system is written as

$$
\begin{aligned}
\dot{\mathbf{e}}^{\mathrm{Re}}= & \Psi \mathbf{x}_{s}^{\mathrm{Re}}(t)+\mathbf{F}^{\mathrm{Re}}\left(\mathbf{x}_{s}(t)\right)-\boldsymbol{\Psi} \mathbf{x}_{m}^{\operatorname{Im}}(t-\tau) \\
& -\mathbf{F}^{\operatorname{Im}}\left(\mathbf{x}_{m}(t-\tau)\right)+\mathbf{L}^{\mathrm{Re}}, \\
\dot{\mathbf{e}}^{\mathrm{Im}}= & \boldsymbol{\Psi} \mathbf{x}_{s}^{\operatorname{Im}}(t)+\mathbf{F}^{\operatorname{Im}}\left(\mathbf{x}_{s}(t)\right)+\boldsymbol{\Psi} \mathbf{x}_{m}^{\mathrm{Re}}(t-\tau) \\
& +\mathbf{F}^{\operatorname{Re}}\left(\mathbf{x}_{m}(t-\tau)\right)+\mathbf{L}^{\operatorname{Im}} .
\end{aligned}
$$

For positive parameters, we may now define a Lyapunov function for this system by the tracking positive definite quantity:

$$
\begin{aligned}
& V(t)=\frac{1}{2}\left[\left(\mathbf{e}^{\mathrm{Re}}\right)^{T} \mathbf{e}^{\mathrm{Re}}+\left(\mathbf{e}^{\operatorname{Im}}\right)^{T} \mathbf{e}^{\mathrm{Im}}\right] \\
& =\frac{1}{2}\left[\left(e_{w_{1}}^{2}+e_{w_{3}}^{2}+\cdots+e_{w_{2 n-1}}^{2}\right)\right. \\
& \left.\quad+\left(e_{w_{2}}^{2}+e_{w_{4}}^{2}+\cdots+e_{w_{2 n}}^{2}\right)\right]=\frac{1}{2}\left(\sum_{h=1}^{n} e_{w_{2 h-1}}^{2}\right. \\
& \left.+\sum_{h=1}^{n} e_{w_{2 h}}^{2}\right) .
\end{aligned}
$$

Note now that the total time derivative of $V(t)$ along the trajectory of error system (30) is as follows:

$$
\begin{aligned}
& \dot{V}(t)=\left(\dot{\mathbf{e}}^{\mathrm{Re}}\right)^{T} \mathbf{e}^{\mathrm{Re}}+\left(\dot{\mathbf{e}}^{\mathrm{Im}}\right)^{T} \mathbf{e}^{\mathrm{Im}}, \\
& =\left(\boldsymbol{\Psi} \mathbf{x}_{s}^{\mathrm{Re}}(t)+\mathbf{F}\left(\mathbf{x}_{s}^{\mathrm{Re}}(t)\right)-\boldsymbol{\Psi} \mathbf{x}_{m}^{\operatorname{Im}}(t-\tau)\right. \\
& \left.\quad-\mathbf{F}\left(\mathbf{x}_{m}^{\operatorname{Im}}(t-\tau)\right)+\mathbf{L}^{\mathrm{Re}}\right)^{T} \mathbf{e}^{\mathrm{Re}}+\left(\boldsymbol{\Psi} \mathbf{x}_{s}^{\operatorname{Im}}(t)\right. \\
& \quad+\mathbf{F}\left(\mathbf{x}_{s}^{\operatorname{Im}}(t)\right)+\boldsymbol{\Psi} \mathbf{x}_{m}^{\mathrm{Re}}(t-\tau)+\mathbf{F}\left(\mathbf{x}_{m}^{\mathrm{Re}}(t-\tau)\right) \\
& \left.\quad+\mathbf{L}^{\operatorname{Im}}\right)^{T} \mathbf{e}^{\operatorname{Im}} .
\end{aligned}
$$

By substituting from (26) about $\mathbf{L}^{\mathrm{Re}}$ and $\mathbf{L}^{\mathrm{Im}}$ in (32) we obtain

$$
\begin{aligned}
\dot{V}(t) & =-\xi\left[\left(\mathbf{e}^{\mathrm{Re}}\right)^{T} \mathbf{e}^{\mathrm{Re}}+\left(\mathbf{e}^{\mathrm{Im}}\right)^{T} \mathbf{e}^{\mathrm{Im}}\right], \\
& =-\xi\left(\sum_{h=1}^{n} e_{w_{2 h-1}}^{2}+\sum_{h=1}^{n} e_{w_{2 h}}^{2}\right) .
\end{aligned}
$$

Obviously, $V(t)$ is a positive definite capacity and its subordinate is negatively definite; therefore as per the Lyapunov hypothesis, intricate mistake system (29) is asymptotically steady, which implies that $e_{w_{2 h}} \rightarrow 0$ and $e_{w_{2 h-1}} \rightarrow 0$ as $t \rightarrow \infty$ and $h=1,2, \ldots, n$. Along these lines, the CALS between systems (22) and (23) is accomplished. This supplements the confirmation.

At last, by applying it for two comparable riotous complexes Lü systems in Section 5, our composition is stated.

\section{Example}

5.1. Construction of the Controller. In the current subsection, we can demonstrate the likelihood and effectiveness of the proposed synchronization plot in the forward segment. We review the CALS of two indistinguishable chaotic complex Lü systems. The main and the slave systems are consequently defined, individually, as follows:

$$
\begin{aligned}
& \dot{x}_{m}=\rho\left(y_{m}-x_{m}\right), \\
& \dot{y}_{m}=v y_{m}-x_{m} z_{m}, \\
& \dot{z}_{m}=\frac{1}{2}\left(\bar{x}_{m} y_{m}+x_{m} \bar{y}_{m}\right)-\mu z_{m}+j w_{2 m} w_{3 m}, \\
& \dot{x}_{s}=\rho\left(y_{s}-x_{s}\right)+L_{1}, \\
& \dot{y}_{s}=v y_{s}-x_{s} z_{s}+L_{2}, \\
& \dot{z}_{s}=\frac{1}{2}\left(\bar{x}_{s} y_{s}+x_{s} \bar{y}_{s}\right)-\mu z_{s}+j w_{2 s} w_{3 s}+L_{3},
\end{aligned}
$$

where $x_{m}=w_{1 m}+j w_{2 m}, y_{m}=w_{3 m}+j w_{4 m}, z_{m}=w_{5 m}+j w_{6 m}$, $x_{s}=w_{1 s}+j w_{2 s}, y_{s}=w_{3 s}+j w_{4 s}, z_{s}=w_{5 s}+j w_{6 s}, L_{1}=\zeta_{1}+j \zeta_{2}$, 
$L_{2}=\zeta_{3}+j \zeta_{4}$, and $L_{3}=\zeta_{5}+j \zeta_{6}$ are complex control functions, respectively, which are to be determined.

Complex systems (34) and (35) can be formed, respectively, as

$$
\begin{aligned}
& \left(\begin{array}{c}
\dot{x}_{m} \\
\dot{y}_{m} \\
\dot{z}_{m}
\end{array}\right)=\left(\begin{array}{ccc}
-\rho & \rho & 0 \\
0 & v & 0 \\
0 & 0 & -\mu
\end{array}\right)\left(\begin{array}{c}
\dot{x}_{m} \\
\dot{y}_{m} \\
\dot{z}_{m}
\end{array}\right) \\
& +\left(\begin{array}{c}
o \\
-x_{m} z_{m} \\
\frac{1}{2}\left(\bar{x}_{m} y_{m}+x_{m} \bar{y}_{m}\right)+j w_{2 m} w_{3 m}
\end{array}\right) \text {, } \\
& \left(\begin{array}{c}
\dot{x}_{s} \\
\dot{y}_{s} \\
\dot{z}_{s}
\end{array}\right)=\left(\begin{array}{ccc}
-\rho & \rho & 0 \\
0 & v & 0 \\
0 & 0 & -\mu
\end{array}\right)\left(\begin{array}{c}
\dot{x}_{s} \\
\dot{y}_{s} \\
\dot{z}_{s}
\end{array}\right) \\
& +\left(\begin{array}{c}
o \\
-x_{s} z_{s} \\
\frac{1}{2}\left(\bar{x}_{s} y_{s}+x_{s} \bar{y}_{s}\right)+j w_{2 s} w_{3 s}
\end{array}\right)
\end{aligned}
$$

So, by comparing complex systems (36) and (37) with the form of systems (22) and (23), respectively, we find

$$
\begin{aligned}
\Psi & =\left(\begin{array}{ccc}
-\rho & \rho & 0 \\
0 & v & 0 \\
0 & 0 & -\mu
\end{array}\right), \\
\mathbf{F}\left(\mathbf{x}_{m}\right) & =\left(\begin{array}{c}
o \\
\frac{1}{2}\left(\bar{x}_{m} y_{m}+x_{m} \bar{y}_{m}\right)+j w_{2 m} w_{3 m}
\end{array}\right), \\
\mathbf{F}\left(\mathbf{x}_{s}\right) & =\left(\begin{array}{c}
o \\
\frac{1}{2}\left(\bar{x}_{s} y_{s}+x_{s} \bar{y}_{s}\right)+j w_{2 s} w_{3 s}
\end{array}\right) .
\end{aligned}
$$

According to Theorem 8 , the controller is computed as

$$
\begin{gathered}
L=\left[-\boldsymbol{\Psi} \mathbf{x}_{s}(t)-\mathbf{F}\left(\mathbf{x}_{s}(t)\right)\right]-j\left[\mathbf{\Psi} \mathbf{x}_{m}(t-\tau)+\mathbf{F}\left(\mathbf{x}_{m}(t-\tau)\right)\right]-\xi \mathbf{e}, \\
\left(\begin{array}{c}
L_{1} \\
L_{2} \\
L_{3}
\end{array}\right)=\left(\begin{array}{c}
-\rho\left(y_{s}(t)-x_{s}(t)\right)-j \rho\left(y_{m}(t-\tau)-x_{m}(t-\tau)\right)-\xi e_{1} \\
-v y_{s}(t)+\varphi_{1}-j\left(v y_{m}(t-\tau)-\varphi_{2}\right)-\xi e_{2} \\
-\varphi_{3}+\mu z_{s}(t)-j w_{2 s}(t) w_{3 s}(t)-j\left(\varphi_{4}-\mu z_{m}(t-\tau)+j w_{2 m}(t-\tau) w_{3 m}(t-\tau)\right)-\xi e_{3}
\end{array}\right),
\end{gathered}
$$

where $\varphi_{1}=x_{s}(t) z_{s}(t), \varphi_{2}=x_{m}(t-\tau) z_{m}(t-\tau), \varphi_{3}=$ $1 / 2\left(\bar{x}_{s}(t) y_{s}(t)+x_{s}(t) \bar{y}_{s}(t)\right)$, and $\varphi_{4}=1 / 2\left(\bar{x}_{m}(t-\tau) y_{m}(t-\right.$ $\left.\tau)+x_{m}(t-\tau) \bar{y}_{m}(t-\tau)\right)$.
So, the controller in (39) can be written as

$$
\begin{aligned}
\left(\begin{array}{c}
\zeta_{1}+j \zeta_{2} \\
\zeta_{3}+j \zeta_{4} \\
\zeta_{5}+j \zeta_{6}
\end{array}\right)= & \left(\begin{array}{c}
-\rho\left(w_{3 s}(t)-w_{1 s}(t)-w_{4 m}(t-\tau)+w_{2 m}(t-\tau)\right)-\xi e_{w_{1}} \\
-v\left(w_{3 s}(t)-w_{4 m}(t-\tau)\right)+\varphi_{5}-\xi e_{w 3} \\
-\varphi_{7}+\mu\left(w_{5 s}(t)-w_{6 m}(t-\tau)\right)+w_{2 m}(t-\tau) w_{3 m}(t-\tau)-\xi e_{w 5}
\end{array}\right) \\
& +j\left(\begin{array}{c}
-\rho\left(w_{4 s}(t)-w_{2 s}(t)+w_{3 m}(t-\tau)-w_{1 m}(t-\tau)\right)-\xi e_{w_{2}} \\
-v\left(w_{4 s}(t)+w_{3 m}(t-\tau)\right)+\varphi_{6}-\xi e_{w 4} \\
-\varphi_{8}+\mu\left(w_{6 s}(t)+w_{5 m}(t-\tau)\right)-w_{2 s}(t) w_{3 s}(t)-\xi e_{w 6}
\end{array}\right)
\end{aligned}
$$

where $\varphi_{5}=w_{1 s}(t) w_{5 s}(t)-w_{2 s}(t) w_{6 s}(t)-w_{1 m}(t-\tau) w_{6 m}(t-$ $\tau)-w_{2 m}(t-\tau) w_{5 m}(t-\tau), \varphi_{6}=w_{1 s}(t) w_{6 s}(t)+w_{2 s}(t) w_{5 s}(t)+$ $w_{1 m}(t-\tau) w_{5 m}(t-\tau)-w_{2 m}(t-\tau) w_{6 m}(t-\tau), \varphi_{7}=w_{1 s}(t) w_{3 s}(t)+$ $w_{2 s}(t) w_{4 s}(t), \varphi_{8}=w_{1 m}(t-\tau) w_{3 m}(t-\tau)+w_{2 m}(t-\tau) w_{4 m}(t-\tau)$.
5.2. Numerical Results. To demonstrate and confirm the value of the suggested plot, we clarify the recreation consequences of the CALS among two indistinguishable chaotic complex systems (34) and (35). Systems (34) and (35) with the 


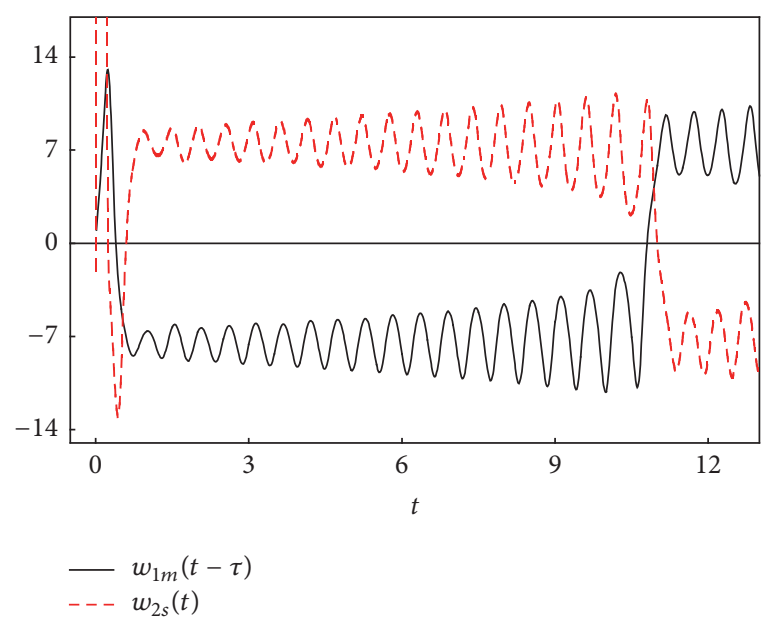

(a)

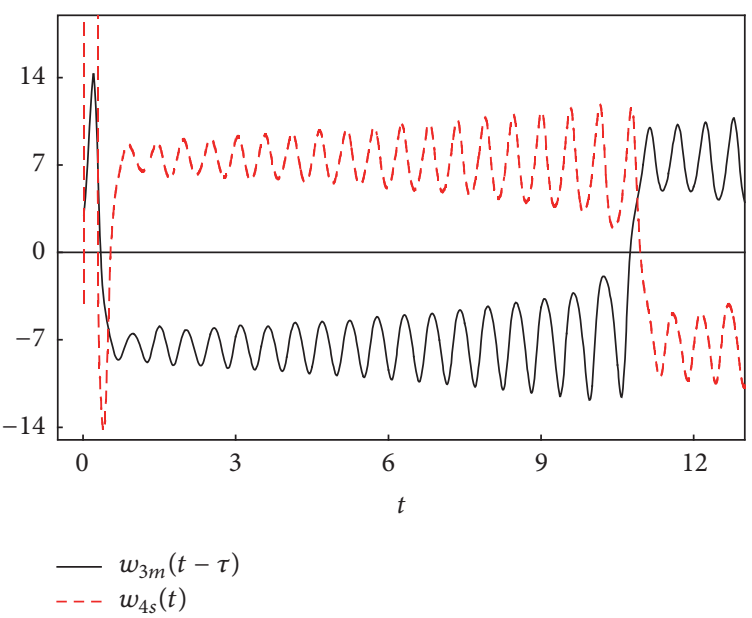

(c)

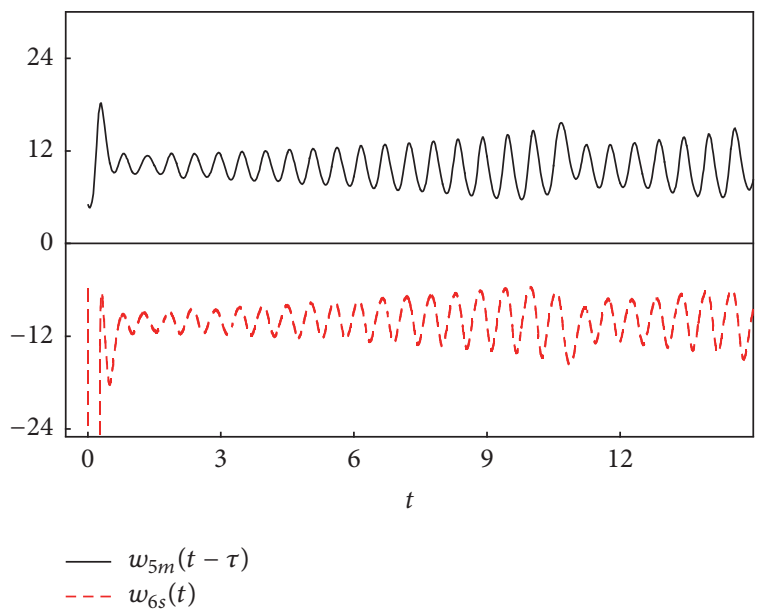

(e)

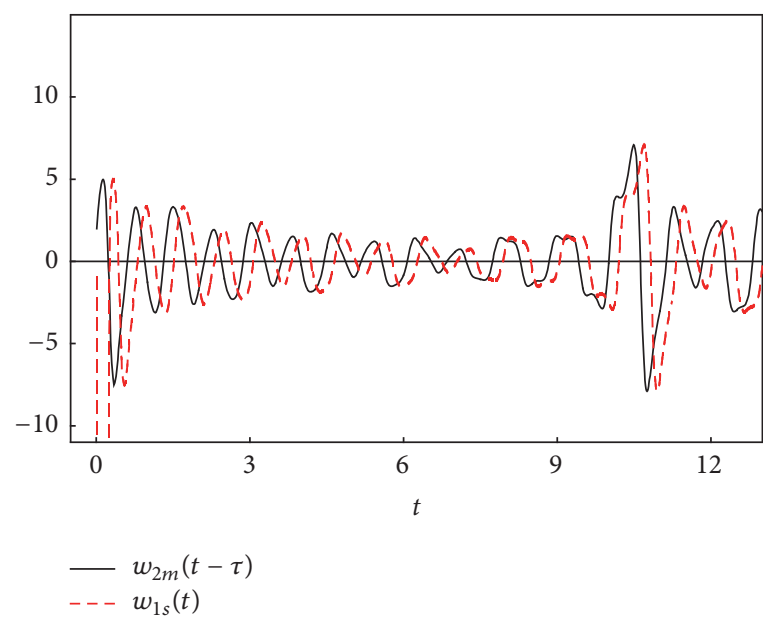

(b)

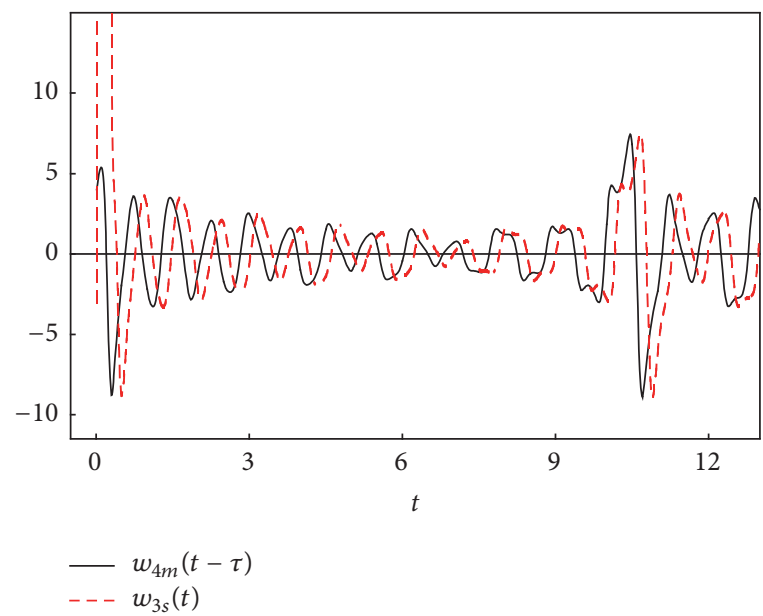

(d)

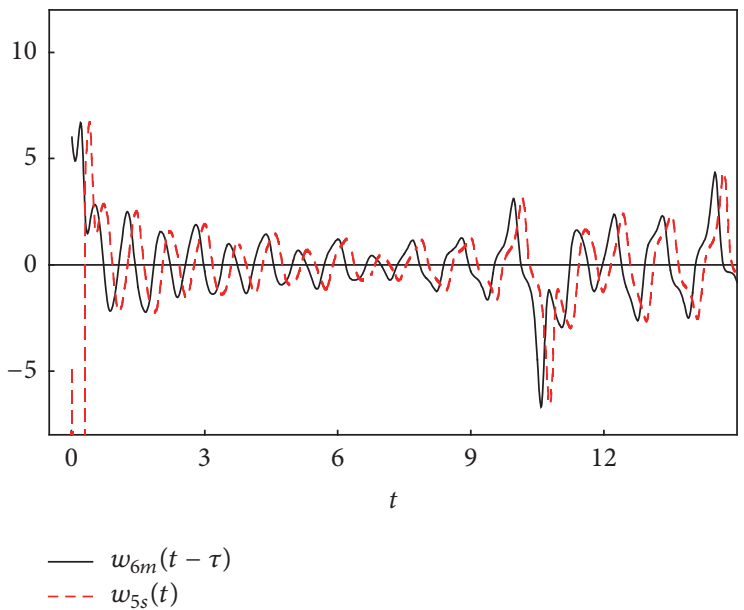

(f)

Figure 4: CALS between two identical systems (34) and (35) with the controller (40). (a) $w_{1 m}(t-\tau)$ and $w_{2 s}(t)$. (b) $w_{2 m}(t-\tau)$ and $w_{1 s}(t)$. (c) $w_{3 m}(t-\tau)$ and $w_{4 s}(t)$. (d) $w_{4 m}(t-\tau)$ and $w_{3 s}(t)$. (e) $w_{5 m}(t-\tau)$ and $w_{6 s}(t)$. (f) $w_{6 m}(t-\tau)$ and $w_{5 s}(t)$.

controller (40) are illuminated numerically, and the parameters are picked as $\rho=23, \mu=6$, and $v=10$. The underlying necessity of the main model and the underlying state of the slave models $\xi, \tau$ are considered basically $\left(x_{m}(0), y_{m}(0)\right.$,
$\left.z_{m}(0)\right)^{T}=(1+2 j, 3+4 j, 5+6 j)^{T},\left(x_{s}(0), y_{s}(0), z_{s}(0)\right)^{T}=$ $(-1-2 j,-3-4 j,-5-6 j)^{T}, \xi=10$, and $\tau=0.2$.

In Figure 4 the arrangements of (34) and (35) are plotted which are subject to different beginning conditions and 


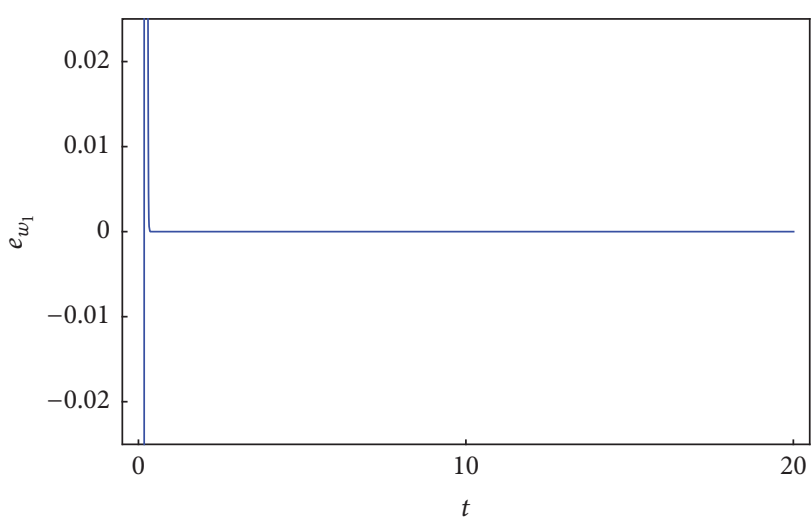

(a)

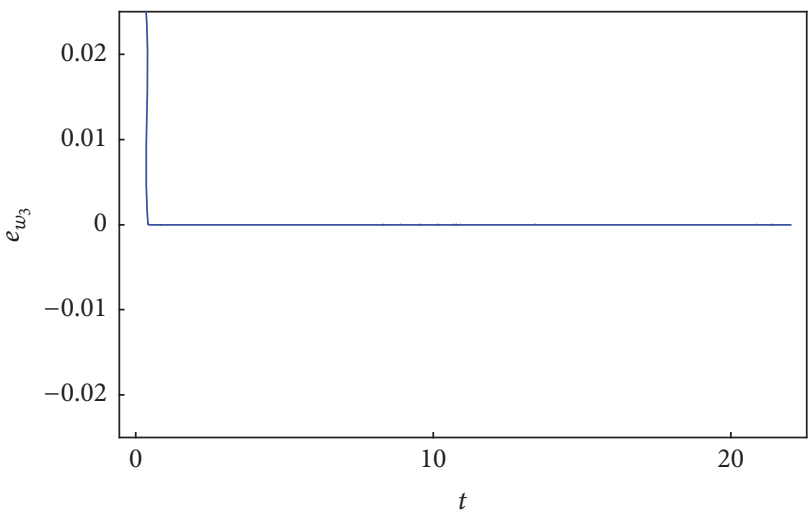

(c)

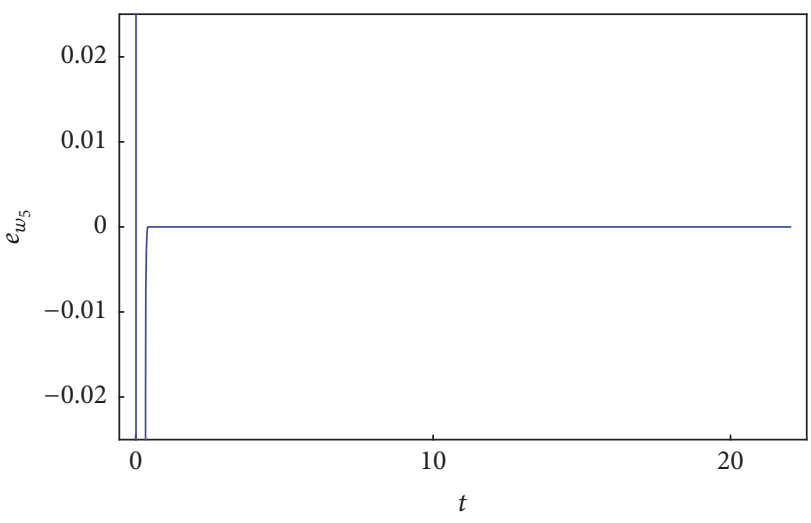

(e)

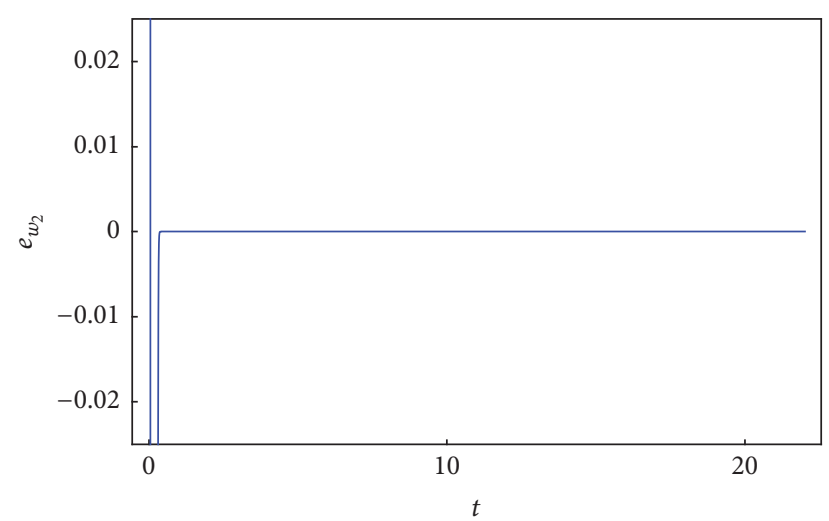

(b)

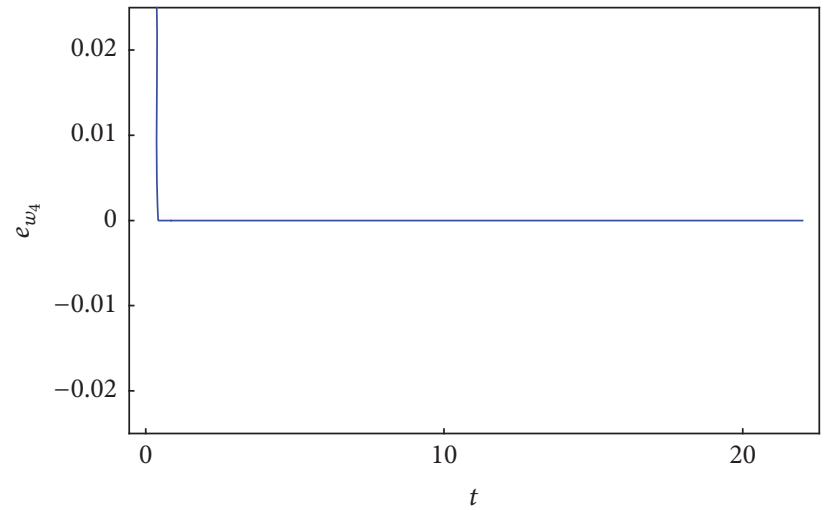

(d)

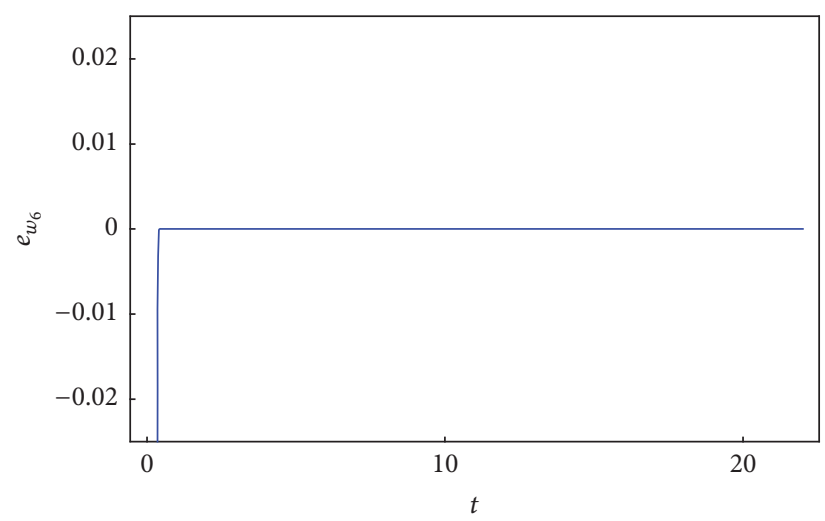

(f)

FIGURE 5: CALS errors between systems (34) and (35): (a) $w_{1 s}(t)-w_{2 m}(t-\tau)$. (b) $w_{2 s}(t)+w_{1 m}(t-\tau)$. (c) $w_{3 s}(t)-w_{4 m}(t-\tau)$. (d) $w_{4 s}(t)+w_{3 m}(t-\tau)$. (e) $w_{5 s}(t)-w_{6 m}(t-\tau)$. (f) $w_{6 s}(t)+w_{5 m}(t-\tau)$.

demonstrate that CALS is to be surely accomplished after a next to no time $t$. We can see that $w_{1 s}, w_{3 s}, w_{5 s}$ have a similar indication of $w_{2 m}, w_{4 m}, w_{6 m}$ with time lag, while $w_{2 s}, w_{4 s}, w_{6 s}$ have an inverse indication of $w_{1 m}, w_{3 m}, w_{5 m}$ with time lag. This implies that LS is accomplished between the imaginary part of system (34) and the real part of system (35) while ALS happens between the real part of slave system (34) and the fanciful part of the main system (35). It is clear from Figure 4 that the state variables of the main system synchronize with different state variables of the slave system. In this way, the CALS gives more prominent security in secure communications. Figure 4 indicates that CALS is accomplished after little time interim. The CALS errors are plotted in Figure 5. Of course from the above exschemeatory contemplations the CALS errors $e_{w_{2 h-1}}$ and $e_{w_{2 h}}$ converge to zero as $t \rightarrow \infty$ and $h=1,2,3$. In Figure 5 it can be seen that the errors will approach zero after little estimation of $t$. The development of the attractors of main and slave systems in the wake of accomplishing the CALS is shown in Figure 6. Another marvel is represented in Figure 6 and does not model 


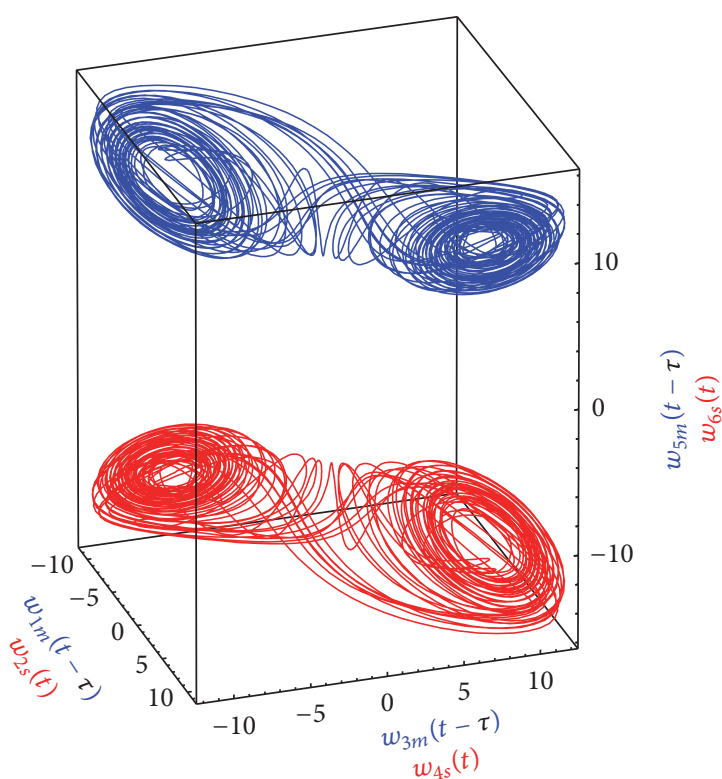

(a)

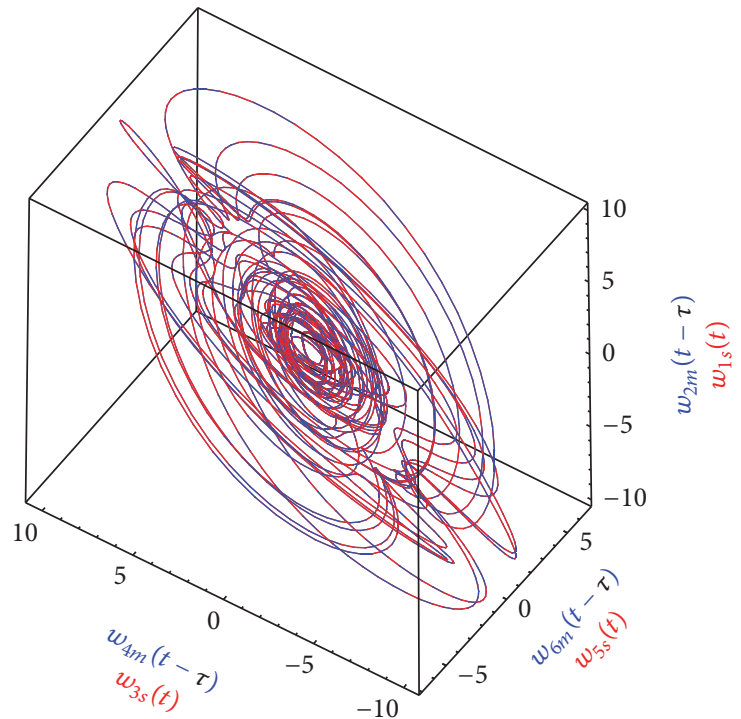

(b)

Figure 6: The attractors of systems (34) and (35). (a) Attractor of the main system has the opposite shape of the slave system. (b) Attractor of the main system has the similar shape of the slave system.

up in a wide range of synchronizations in the writing. The attractors of the main and slave systems in CALS are moving in opposite or similar shape to each other with different state variables and time lag as found in Figure 6. In Figure 6(a), the attractor of the main system in $\left(w_{1 m}(t-\tau), w_{3 m}(t-\right.$ $\left.\tau), w_{5 m}(t-\tau)\right)$ has the opposite shape of the slave system in $\left(w_{2 s}(t), w_{4 s}(t), w_{6}(t)\right)$, while the attractor of the slave system in $\left(w_{1 s}(t), w_{3 s}(t), w_{5}(t)\right)$ has the similar shape of the main system with time lag in $\left(w_{2 m}(t-\tau), w_{4 m}(t-\tau), w_{6 m}(t-\tau)\right)$ as shown in Figure 6(b).

In the numerical reenactments, we register the module errors and phase errors of main and slave models, respectively. For every unpredictable number, the module and phase are resolved as follows:

$$
\begin{aligned}
& \rho_{x}=\sqrt{\left(x^{\mathrm{Re}}\right)^{2}+\left(x^{\mathrm{Im}}\right)^{2},} \\
& \theta_{x}= \begin{cases}\arctan \left(\frac{x^{\mathrm{Im}}}{x^{\mathrm{Re}}}\right), & x^{\mathrm{Re}}>0, x^{\mathrm{Im}} \geqslant 0, \\
2 \pi+\arctan \left(\frac{x^{\mathrm{Im}}}{x^{\mathrm{Re}}}\right), & x^{\mathrm{Re}}>0, x^{\mathrm{Im}}<0, \\
\pi+\arctan \left(\frac{x^{\mathrm{Im}}}{x^{\mathrm{Re}}}\right), & x^{\mathrm{Re}}<0 .\end{cases}
\end{aligned}
$$

Figure 7 models the modules errors and phases errors of main system (34) and slave systems (35). It is clear from Figures 7(a), 7(b), and 7(c) that the modules errors $\rho_{x_{m}}-\rho_{x_{s}}, \rho_{y_{m}}-\rho_{y_{s}}$, and $\rho_{z_{z}}-\rho_{z}$ converge to zero as $t \rightarrow \infty$, while the phases errors $\theta_{x_{m}}-\theta_{x_{s}}, \theta_{y_{m}}-\theta_{y_{s}}$, and $\theta_{z_{m}}-\theta_{z_{s}}$ go to $\pi / 2$ or $-\pi / 2$ as $t \rightarrow \infty$; see Figures $7(\mathrm{~d}), 7(\mathrm{e})$, and $7(\mathrm{f})$.

\section{Conclusions}

In this paper, we have presented a cutting edge chaotic complex Lü system (2) and we concentrated the elements of it. By affixing an unpredictable expression of the third condition of the complex Lü system (1), we can develop this system. In this system all factors are intricate. Our system in real adaptation is six measurements or by different words system (2) are high measurements. In spite of this, our new system does not create hyperchaotic practices. Consequently, we close from these outcomes that the expansion in the measurements is the important and not sufficient condition for producing hyperchaotic practices. The steadiness investigations of the fixed purposes of system (2) are completed. We presented in Section 3 different types of chaotic complex Lü systems which can be comparatively explored as we accomplished for system (2) in Section 2. These new systems leave room for further investigations.

We present another kind of complex synchronization which is called complex antilag synchronization (CALS). A definition of CALS of two indistinguishable chaotic complex systems is given. We dissect and concentrate the CALS concerning two indistinguishable chaotic complex nonlinear systems. The CALS can be concentrated just in complex nonlinear systems. The CALS can be considered as syncretizing among ALS and LS (see Figure 4). LS occurs between a real part of the slave system and an imaginary part of the main system, while ALS occurs between the real part of the main system and the fanciful part of a slave system. In CALS, the state variables of the main system synchronize with different state variables of the slave system (see Figure 4). Thus, CALS gives more prominent security in secure interchanges. The 


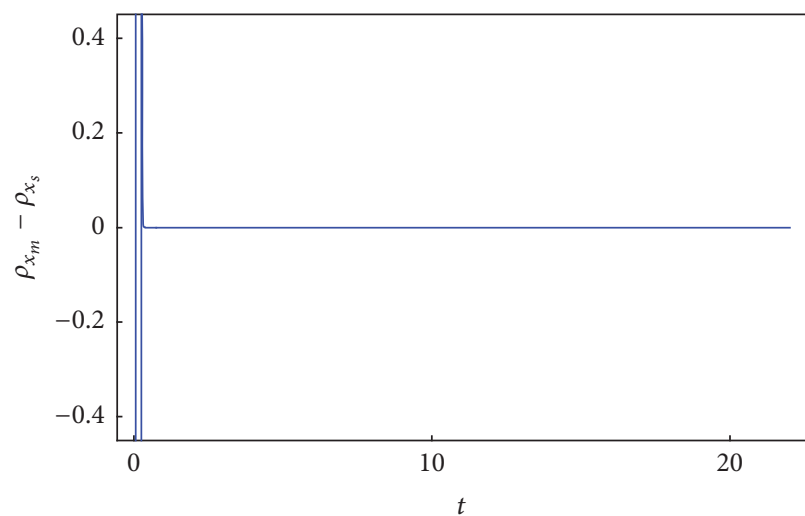

(a)

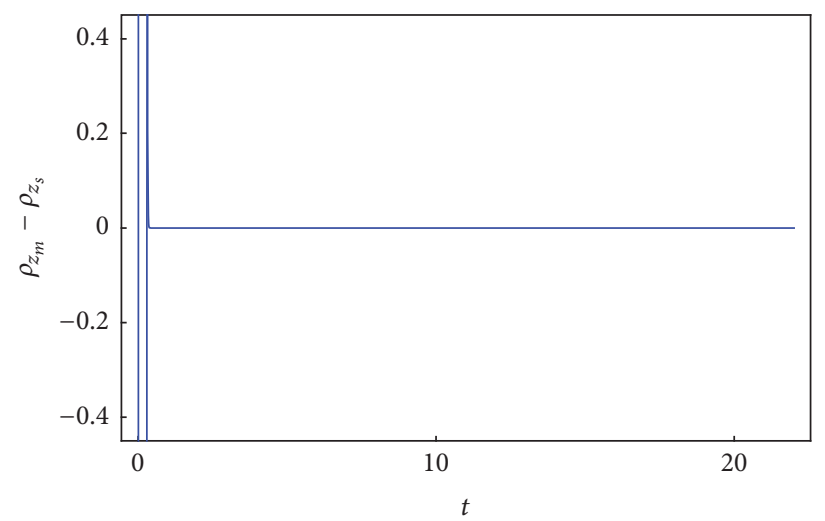

(c)

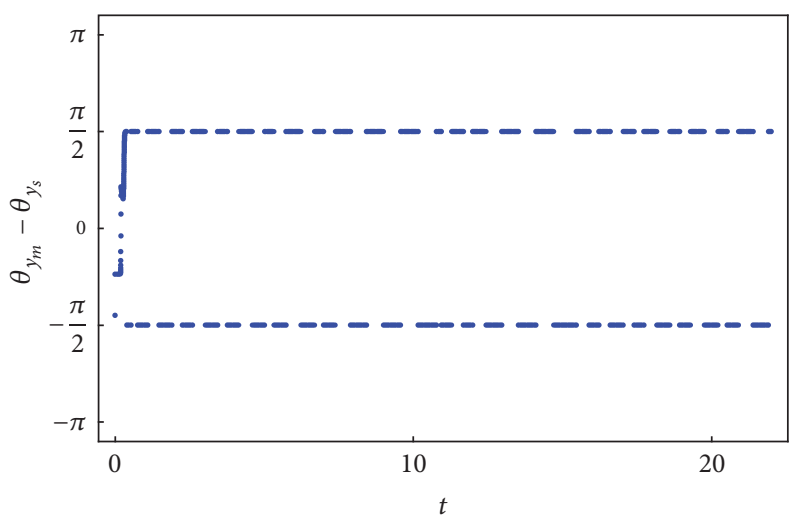

(e)

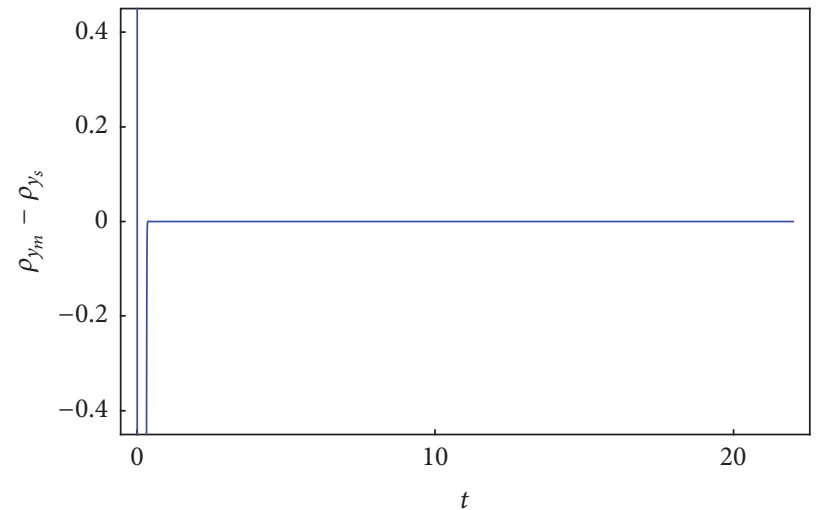

(b)

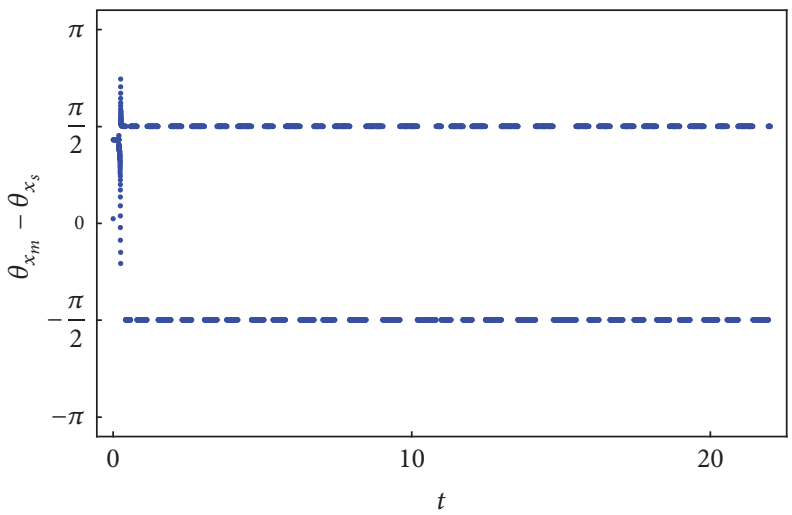

(d)

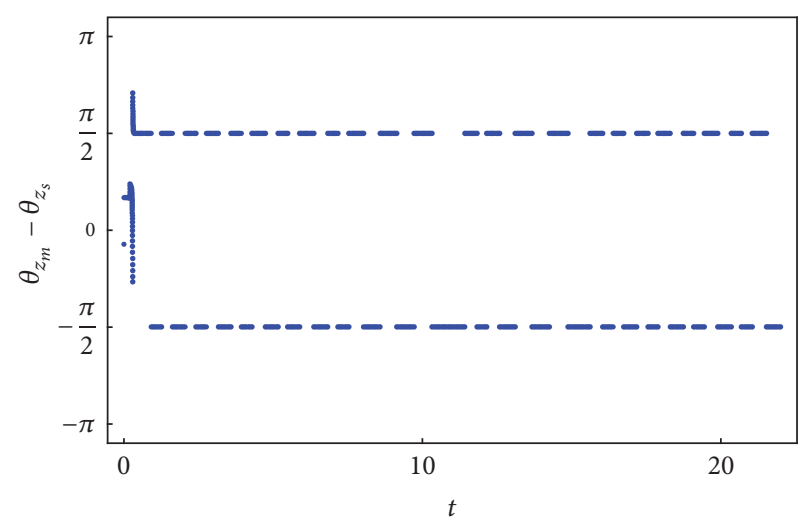

(f)

FIGURE 7: The modules errors and phases errors of systems (34) and (35): (a) $\rho_{x_{m}}-\rho_{x_{s}}$ versus $t$. (b) $\rho_{y_{m}}-\rho_{y_{s}}$ versus $t$. (c) $\rho_{z_{m}}-\rho_{z_{s}}$ versus $t$. (d) $\theta_{x_{m}}-\theta_{x_{s}}$ versus $t$. (e) $\theta_{y_{m}}-\theta_{y_{s}}$ versus $t$. (f) $\theta_{z_{m}}-\theta_{z_{s}}$ versus $t$.

most outstanding normal for the CALS is the attractors of the main and slave systems are moving in opposite or similar shape to each other with different state variables (see Figures 6(a) and 6(b)). These marvels did not come to pass and appear for any sorts of synchronization in the writing.

A scheme is laid out to acknowledge CALS of two indistinguishable chaotic complex nonlinear systems in view of Lyapunov capacities. Amid this scheme, we analytically concluded the control complex capacities to accomplish
CALS. It is simple and advantageous to utilize this scheme for chaotic and hyperchaotic complex systems. We apply our scheme, for instance, for two indistinguishable chaotic complex systems with different introductory qualities, main system (34) and slave systems (35). All the hypothetical outcomes are verified by numerical recreations of our case. A great assertion is found as shown in Figures 4, 5, and 6. In Figure 7 we register the modules errors and phase errors in light of the fact that, in the complex nonlinear 
dynamical systems, the noticeable or quantifiable physical amounts more often than not are module and phase.

\section{Conflicts of Interest}

The authors declare that there are no conflicts of interest regarding the publication of this paper.

\section{References}

[1] T. K. Alligood, T. Sauer, and J. A. Yorke, An Introduction to Dynamical Systems, Chaos, Springer, New York, NY, USA, 1997.

[2] E. N. Lorenz, "Deterministic nonperiodic flow," Journal of the Atmospheric Sciences, vol. 20, no. 2, pp. 130-141, 1963.

[3] F. J. Li, N. Li, Y. P. Liu, and Y. Gan, "Linear and nonlinear generalized synchronization of a class of chaotic systems by using a single driving variable," Journal of Applied Physical Science, vol. 58, pp. 779-784, 2009.

[4] Y. X. Wang and M. J. Meng, "Chaotic control of the coupled logistic map," Acta Physica Sinica, vol. 57, pp. 726-773.

[5] S. Liu, B. Liu, and P. M. Shi, "Nonlinear feedback control of Hopf bifurcation in a relative rotation dynamical system," Acta Physica Sinica, vol. 58, no. 7, pp. 4383-4389, 2009.

[6] J. Lü and G. Chen, "A new chaotic attractor coined," International Journal of Bifurcation and Chaos in Applied Sciences and Engineering, vol. 12, no. 3, pp. 659-661, 2002.

[7] C. Liu, T. Liu, L. Liu, and K. Liu, "A new chaotic attractor," Chaos, Solitons \& Fractals, vol. 22, no. 5, pp. 1031-1038, 2004.

[8] A. C. Fowler, J. D. Gibbon, and M. J. McGuinness, "The complex Lorenz equations," Physica D, vol. 4, no. 2, pp. 139-163, 1982.

[9] G. M. Mahmoud, M. A. Al-Kashif, and S. A. Aly, "Basic properties and chaotic synchronization of complex Lorenz system," International Journal of Modern Physics C, vol. 18, no. 2, pp. 253-265, 2007.

[10] A. Rauh, L. Hannibal, and N. B. Abraham, "Global stability properties of the complex Lorenz model," Physica D, vol. 99, no. 1, pp. 45-58, 1996.

[11] J. H. Peng, E. J. Ding, M. Ding, and W. Yang, "Synchronizing hyperchaos with a scalar transmitted signal," Physical Review Letters, vol. 76, no. 6, pp. 904-907, 1996.

[12] G. M. Mahmoud, T. Bountis, and E. E. Mahmoud, "Active control and global synchronization of the complex Chen and Lü systems," International Journal of Bifurcation and Chaos in Applied Sciences and Engineering, vol. 17, no. 12, pp. 4295-4308, 2007.

[13] E. E. Mahmoud, "Complex complete synchronization of two nonidentical hyperchaotic complex nonlinear systems," Mathematical Methods in the Applied Sciences, vol. 37, no. 3, pp. 321328, 2014.

[14] E. E. Mahmoud and K. M. Abualnaja, "Complex lag synchronization of two identical chaotic complex nonlinear systems," Central European Journal of Physics, vol. 12, no. 1, pp. 63-69, 2014.

[15] Z. Wu, J. Duan, and X. Fu, "Complex projective synchronization in coupled chaotic complex dynamical systems," Nonlinear Dynamics, vol. 69, no. 3, pp. 771-779, 2012.

[16] G. M. Mahmoud and E. E. Mahmoud, "Complex modified projective synchronization of two chaotic complex nonlinear systems," Nonlinear Dynamics, vol. 73, no. 4, pp. 2231-2240, 2013.
[17] F.-F. Zhang, S.-T. Liu, and W.-Y. Yu, "Modified projective synchronization with complex scaling factors of uncertain real chaos and complex chaos," Chinese Physics B, vol. 22, no. 12, article 120505, 2013.

[18] J. Liu, S. Liu, and W. Li, "Complex modified generalized projective synchronization of fractional-order complex chaos and real chaos," Advances in Difference Equations, pp. 1-16, 2015.

[19] J. Liu, "Complex modified hybrid projective synchronization of different dimensional fractional-order complex chaos and real hyper-chaos," Entropy, vol. 16, no. 12, pp. 6195-6211, 2014.

[20] S. Wang, X. Wang, and B. Han, "Complex generalized synchronization and parameter identification of nonidentical nonlinear complex systems," PLoS ONE, vol. 11, no. 3, article e0152099, 2016.

[21] S. Wang, X. Wang, Y. Zhou, and B. Han, "A memristor-based hyperchaotic complex Lü system and its adaptive complex generalized synchronization," Entropy, vol. 18, no. 2, article 58, 2016.

[22] J. Liu, S. Liu, and C. Yuan, "Adaptive complex modified projective synchronization of complex chaotic (hyperchaotic) systems with uncertain complex parameters," Nonlinear Dynamics, vol. 79, no. 2, pp. 1035-1047, 2015.

[23] J. Liu, S. Liu, and F. Zhang, "A novel four-wing hyperchaotic complex system and its complex modified hybrid projective synchronization with different dimensions," Abstract and Applied Analysis, pp. 1-16, 2014.

[24] J. Liu and S. Liu, "Complex modified function projective synchronization of complex chaotic systems with known and unknown complex parameters," Applied Mathematical Modelling, vol. 48, pp. 440-450, 2017.

[25] J. Liu, S. Liu, and J. C. Sprott, "Adaptive complex modified hybrid function projective synchronization of different dimensional complex chaos with uncertain complex parameters," Nonlinear Dynamics, vol. 83, no. 1-2, pp. 1109-1121, 2016.

[26] G. M. Mahmoud and E. E. Mahmoud, "Lag synchronization of hyperchaotic complex nonlinear systems," Nonlinear Dynamics, vol. 67, no. 2, pp. 1613-1622, 2012.

[27] E. E. Mahmoud, "Adaptive anti-lag synchronization of two identical or non-identical hyperchaotic complex nonlinear systems with uncertain parameters," Journal of the Franklin Institute, vol. 349, no. 3, pp. 1247-1266, 2012.

[28] E. E. Mahmoud, "Lag synchronization of hyperchaotic complex nonlinear systems via passive control," Applied Mathematics \& Information Sciences, vol. 7, no. 4, pp. 1429-1436, 2013.

[29] G. M. Mahmoud and E. E. Mahmoud, "Modified projective lag synchronization of two nonidentical hyperchaotic complex nonlinear systems," International Journal of Bifurcation and Chaos, vol. 21, no. 8, pp. 2369-2379, 2011.

[30] M. S. Klamkin and A. Liu, "Three more proofs of routh's theorem," Crux Math, vol. 7, pp. 199-203, 1981.

[31] A. Wolf, J. B. Swift, H. L. Swinney, and J. A. Vastano, "Determining Lyapunov exponents from a time series," Physica D, vol. 16, no. 3, pp. 285-317, 1985.

[32] P. Frederickson, J. L. Kaplan, E. D. Yorke, and J. A. Yorke, "The Liapunov dimension of strange attractors," Journal of Differential Equations, vol. 49, no. 2, pp. 185-207, 1983.

[33] E. E. Mahmoud and M. A. Al-Adwani, "Dynamical behaviors, control and synchronization of a new chaotic model with complex variables and cubic nonlinear terms," Results in Physics, vol. 7, pp. 1346-1356, 2017. 


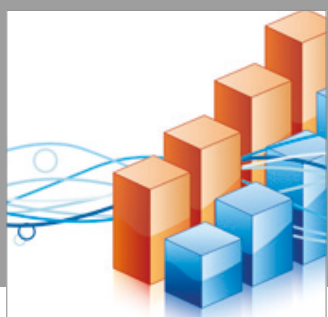

Advances in

Operations Research

vatersals

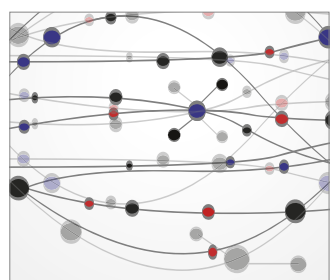

\section{The Scientific} World Journal
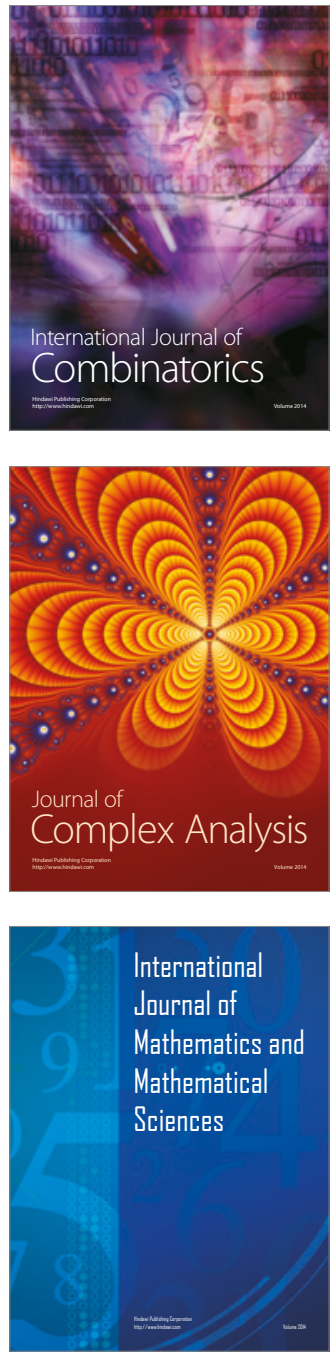
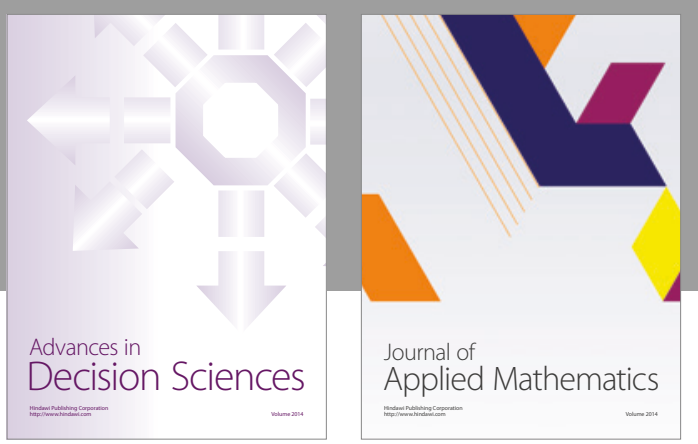

Algebra

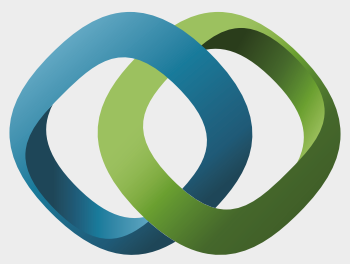

\section{Hindawi}

Submit your manuscripts at

https://www.hindawi.com
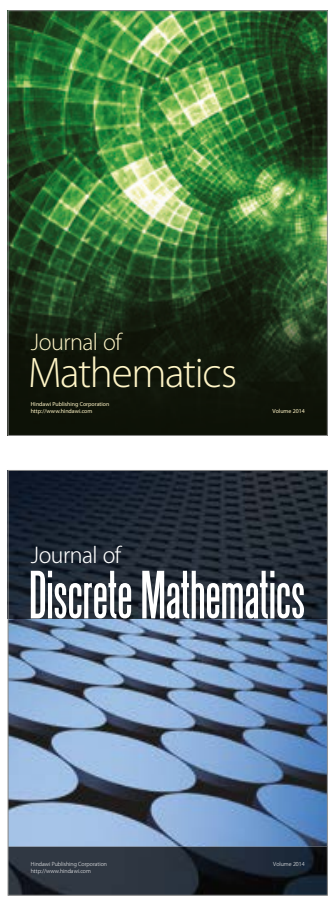

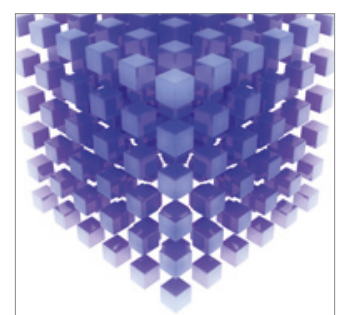

Mathematical Problems in Engineering
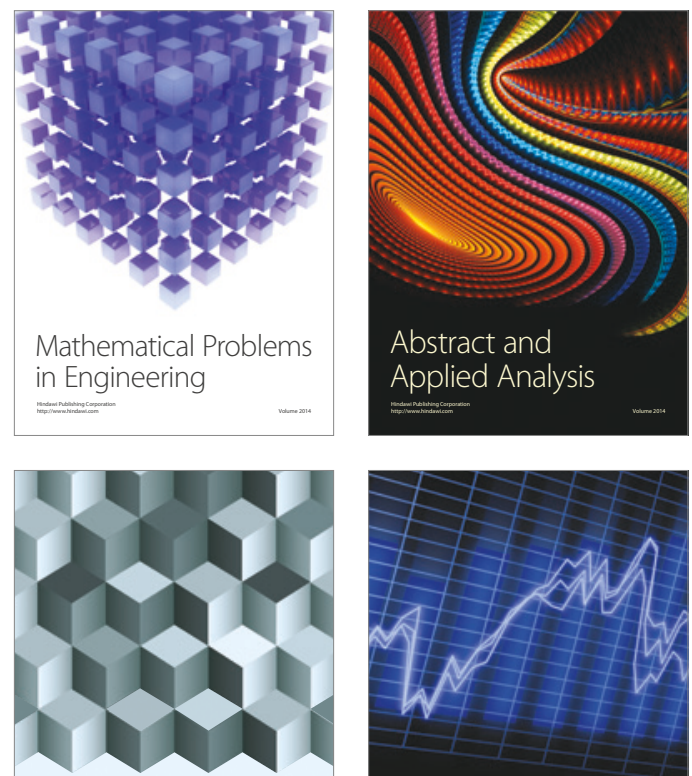

Journal of

Function Spaces

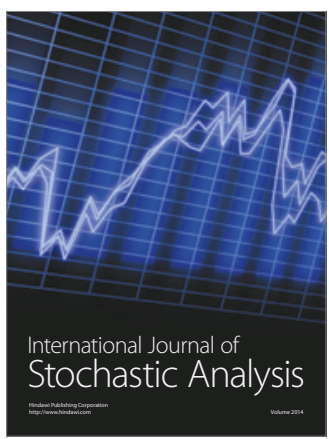

Probability and Statistics
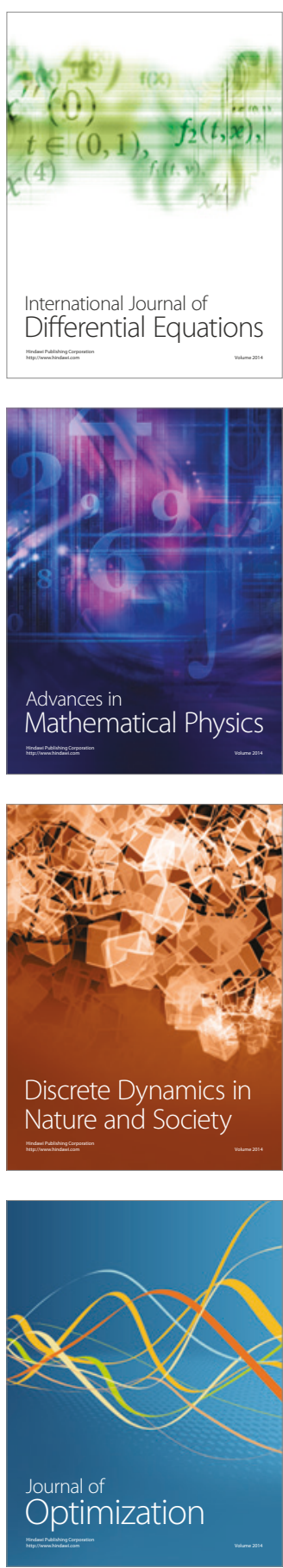